\title{
A splitting result for the free loop space of spheres and projective spaces
}

\author{
Marcel Bökstedt \& Iver Ottosen
}

May 17, 2004

\begin{abstract}
Let $X$ be a 1-connected compact space such that the algebra $H^{*}\left(X ; \mathbb{F}_{2}\right)$ is generated by one single element. We compute the cohomology of the free loop space $H^{*}\left(\Lambda X ; \mathbb{F}_{2}\right)$ including the Steenrod algebra action. When $X$ is a projective space $\mathbb{C} \mathrm{P}^{n}, \mathbb{H} \mathrm{P}^{n}$, the Cayley projective plane $\mathbf{C a} \mathrm{P}^{2}$ or a sphere $S^{m}$ we obtain a splitting result for integral and mod two cohomology of the suspension spectrum $\Sigma^{\infty}(\Lambda X)_{+}$. The splitting is in terms of $\Sigma^{\infty} X_{+}$and the Thom spaces $T h(q \tau), q \geq 0$ of the $q$-fold Whitney sums of the tangent bundle $\tau$ over $X$.
\end{abstract}

\section{Introduction}

The main part of this paper is a computation of mod two cohomology of the free loop space on a space $X$, with the property that $H^{*}\left(X, \mathbb{F}_{2}\right)$ is a truncated polynomial algebra.

This calculation has been done by various other methods before, see [Z], $[\mathrm{KY}]$ and $[\mathrm{CC}]$. We want to make four points in order to explain what is new in this paper.

First, we are using a new general method. The idea is to resolve the space $X$ as a degreewise free cosimplicial space, and then apply the functor "free loop space" degreewise. The Bousfield spectral sequence associated to this cosimplicial space gives us a spectral sequence converging to the (co)homology of the free loop space of $X$, at least if $X$ is simply connected.

This spectral sequence is a generalization of the Eilenberg-Moore spectral sequence. Eilenberg-Moore spectral sequences has been applied in this situation before. The main difference from previous work is that the cosimplicial 
space we consider is not the same as the cosimplicial spaces obtained from homotopy pull back squares like

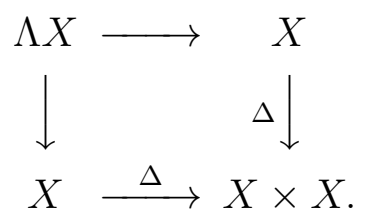

The cosimplicial space we use is related to the cosimplicial resolution of $X$ (in the sense of $[\mathrm{BK}]$ ). In this paper we are using this method to treat some serious cases. From this point of view, we are dealing with test cases for the spectral sequence.

Secondly, the spectral sequence does give us information about the action of the Steenrod algebra on the cohomology of the free loop space. We determine this action for spaces belonging to the following list : $X=S^{n}$, $X=\mathbb{C P}^{n}, X=\mathbb{H} \mathrm{P}^{n}$ and $X=\mathbf{C a} \mathrm{P}^{2}$. Except for the easy sphere case, these results are new.

Thirdly, the circle group acts in an obvious way on a free loop space, and one can consider the Borel construction of this action. The method we consider extends in a natural way (BO1] ) to a spectral sequence converging to the Borel construction. The structures discovered in CS has recently boosted the interest in the homology of the Borel construction. In spite of this, this homology has not been calculated. We intend to study the corresponding spectral sequence. From this point of view, this paper is a preliminary result.

Fourthly, for the spaces in the short list above, we obtain a surprising result. The spaces are all manifolds, and even symmetric spaces. In each case we consider the tangent bundle $\tau$. Let $q \tau$ be the $q$-fold Whitney sum of the tangent bundle with itself. We can consider $q \tau$ as a sub bundle of $(q+1) \tau$. This induces an inclusion of the corresponding Thom spaces, and we define the space $C_{q}(X)$ as the cofiber of the cofibration

$$
T h(q \tau) \rightarrow T h((q+1) \tau) .
$$

The result is that cohomology of $\Sigma^{\infty}\left(\Lambda X_{+}\right)$with coefficients in $\mathbb{F}_{2}$ is the same $\mathcal{A}$-module as the cohomology of

$$
\Sigma^{\infty}\left(X_{+} \vee \bigvee_{q \geq 0} \Sigma^{(m-2)(q+1)} C_{q}(X)\right),
$$

where $m$ denotes the degree of the algebra generator for $H^{*}\left(X ; \mathbb{F}_{2}\right)$.

A splitting discovered by Ziller $[\mathbb{Z}$ shows that the homology with $\mathbb{Z}$ coefficients of the two spectra also agree as abelian groups. 
This strongly suggest the possibility that this wedge is actually homotopy equivalent to $\Sigma^{\infty}(\Lambda X)_{+}$. There is some additional evidence for such a splitting. If $X=S^{n}$ for $n \geq 2$, the spectrum $\Sigma^{\infty}\left(\Lambda X_{+}\right)$is known and simple. It is a wedge of sphere spectra and Moore spectra for the group $\mathbb{Z} / 2$. In fact, in this special case, we do obtain a stable splitting of spectra as above.

Remark 1.1. There are a few simply connected spaces besides the spheres and the projective spaces considered above that have mod two cohomology rings that are truncated polynomial algebras. But there are certain conditions which the cohomology ring of such a space has to satisfy. Let $X$ be a 1connected and compact space. Assume that $H^{*}\left(X ; \mathbb{F}_{2}\right)=\mathbb{F}_{2}[x] /\left(x^{n+1}\right)$ for some $n \geq 1$ and let $m$ be the degree of $x$. If $n \geq 2$ it is known known $\mathrm{A}$, [T] that $m=2,4$ or $m=8$ and $n=2$. For $n=1$ we have that $m$ can be any integer bigger that 2 .

Notation: All cohomology groups are with $\mathbb{F}_{2}$-coefficients unless stated otherwise. We write $\mathcal{A}$ for the mod two Steenrod algebra, $\mathcal{U}$ for the category of unstable $\mathcal{A}$-modules and $\mathcal{K}$ for the category of unstable $\mathcal{A}$-algebras. The degree of an element $x$ is denoted $|x|$. The category of non negatively graded $\mathbb{F}_{2}$-algebras with the property that $a^{2}=a$ if $|a|=0$ is denoted $\mathcal{A l g}$. Note that we have a forgetful functor $\mathcal{K} \rightarrow \mathcal{A l g}$.

We write $\Omega: \mathcal{A l g} \rightarrow \mathcal{A l g}$ for the de Rham complex functor. Thus $\Omega(A)$ for an $A \in \mathcal{A l g}$ means the free $A$-algebra on generators $\{\mathbf{d} x \mid x \in A\}$ of degree $|\mathbf{d} x|=|x|-1$ modulo the relations $\mathbf{d}(x+y)=\mathbf{d} x+\mathbf{d} y, \mathbf{d}(x y)=\mathbf{d}(x) y+x \mathbf{d}(y)$ and $(\mathbf{d} x)^{2}=0$. We always write $\mathbf{d}$ for the de Rham differential in order to distinguish it from the simplicial face maps $d_{i}$. By $s \mathcal{C}$ we mean the category of simplicial objects in the category $\mathcal{C}$.

\section{Results via a simplicial resolution in $s \mathcal{A} l g$}

In this section we compute the $E_{2}$-term of a spectral sequence form BO1] converging toward $H^{*}(\Lambda X)$ in the case where $X$ is a 1-connected, compact space and $H^{*} X$ a truncated polynomial algebra $\mathbb{F}_{2}[x] /\left(x^{n+1}\right)$. The $E_{2}$-term is given by the (non abelian) derived functors of a twisted de Rham complex functor $\bar{\Omega}$ as follows

$$
E_{2}^{-m, t}=H_{m}\left(H^{*} X ; \bar{\Omega}\right)^{t}:=\left(L_{m} \bar{\Omega}\right)\left(H^{*} X\right)^{t} .
$$

See [BO1] Definition 7.1 and sections 5, 6 for the precise definitions.

Remark 1.1 shows that the operation $\lambda(x)=\mathrm{Sq}^{|x|-1} x$ vanish on $H^{*} X$. By BO1] Theorem 6.4, Theorem 7.6 and Proposition 7.3 this implies that

$$
H_{*}\left(H^{*} X ; \bar{\Omega}\right) \cong H_{*}\left(H^{*} X ; \Omega\right),
$$


so it suffices to compute the derived functors of the ordinary de Rham complex functor over the simplicial category $s \mathcal{A} l g$. It turns out that the two cases $n$ even, $n$ odd are essentially different. The result of the computation appears in Theorem [2.5.

Let $T_{n}(x)$ denotes the graded commutative $\mathbb{F}_{2}$-algebra $\mathbb{F}_{2}[x] /\left(x^{n+1}\right)$ where $n \geq 1$ and $|x| \geq 1$.

Theorem 2.1. There is an almost free simplicial resolution $R_{\bullet} \in s \mathcal{A l g}$ of $T_{n}(x)$ as follows: $R_{q}=\mathbb{F}_{2}\left[x, y_{1}, \ldots, y_{q}\right]$ for $q \geq 0$ where $\left|y_{i}\right|=(n+1)|x|$ and $s_{i}(x)=x, d_{i}(x)=x$ for all $i$ and

$$
\begin{aligned}
& s_{i}\left(y_{j}\right)= \begin{cases}y_{j} & , i \geq j \\
y_{j+1} & , i<j\end{cases} \\
& d_{i}\left(y_{j}\right)= \begin{cases}x^{n+1} & , i=0, j=1 \\
y_{j-1} & , i<j, j>1 \\
y_{j} & , i \geq j, j<q \\
0 & , i=q, j=q .\end{cases}
\end{aligned}
$$

Proof. Similar to the proof of Proposition 8.2 in [BO1].

For a simplicial $\mathbb{F}_{2}$-vector space $V$ we write $C_{*}(V)$ for the associated chain complex with $C_{q}(V)=V_{q}$ and differential $\sum_{i=0}^{q} d_{i}$. We write $N_{*}(V)$ for the normalized chain complex with $N_{q}(V)=\cap_{1 \leq i \leq q} \operatorname{ker}\left(d_{i}\right)$ and differential $d_{0}$. Finally, we write $D_{*}(V)$ for the sub complex of $C_{*}(V)$ generated by the degenerate elements $\mathrm{ML}$.

Definition 2.2. The element $\omega_{q} \in \Omega\left(R_{q}\right)$ is defined by $\omega_{q}=\mathbf{d} y_{1} \ldots \mathbf{d} y_{q}$ for $q>0$ and $\omega_{0}=1$. We use the notation

$$
\begin{array}{ll}
\left(\omega_{q}\right)_{i}=\mathbf{d} y_{1} \ldots \widehat{\mathbf{d} y_{i}} \ldots \mathbf{d} y_{q} & \text { for } 1 \leq i \leq q, \\
\left(\omega_{q}\right)_{i, j}=\mathbf{d} y_{1} \ldots \widehat{\mathbf{d} y_{i}} \ldots \widehat{\mathbf{d} y_{j}} \ldots \mathbf{d} y_{q} & \text { for } 1 \leq i<j \leq q .
\end{array}
$$

Define $\alpha_{q}, \beta_{q} \in \Omega\left(R_{q}\right)$ by

$$
\alpha_{q}=\mathbf{d} x \omega_{q} \quad, \quad \beta_{q}=x \omega_{q}+\mathbf{d} x \sum_{i=1}^{q} y_{i}\left(\omega_{q}\right)_{i}
$$

for $q>0$ and $\alpha_{0}=\mathbf{d} x, \beta_{0}=x$.

Lemma 2.3. For $q \geq 0$ one has that $\omega_{q}$ is a cycle in $N_{q} \Omega\left(R_{\bullet}\right)$ for $n$ odd and $\alpha_{q}, \beta_{q}$ are cycles in $N_{q} \Omega\left(R_{\bullet}\right)$ for $n$ even. Furthermore, the elements $\left(\omega_{q+1}\right)_{r}$, $\left(\omega_{q+1}\right)_{j, k}, y_{r}\left(\omega_{q+1}\right)_{j, k}$ lie in $D_{q+1} \Omega\left(R_{\bullet}\right)$ for any $n$ where $1 \leq j<k \leq q+1$ and $1 \leq r \leq q+1$. 
Proof. For $n$ odd we have $d_{0} \omega_{q}=0$ since $d_{0} \mathbf{d} y_{1}=\mathbf{d}\left(x^{n+1}\right)=0, d_{i} \omega_{q}=0$ for $0<i<q$ since $\left(\mathbf{d} y_{i}\right)^{2}=0$ and $d_{q} \omega_{q}=0$ since $d_{q} \mathbf{d} y_{q}=0$. For $n$ even we have $d_{i} \beta_{q}=0$ for all $i$ since

$$
\begin{gathered}
d_{i}\left(x \omega_{q}\right)= \begin{cases}x^{n+1} \mathbf{d} x \omega_{q-1} & , i=0 \\
0 & , i>0,\end{cases} \\
d_{i}\left(y_{j} \mathbf{d} x\left(\omega_{q}\right)_{j}\right)= \begin{cases}x^{n+1} \mathbf{d} x \omega_{q-1} & , i=0 ; j=1 \\
y_{i} \mathbf{d} x \omega_{q-1} & , 0<i<q ; j=i, i+1 \\
0 & , \text { otherwise. }\end{cases}
\end{gathered}
$$

Note that $\mathbf{d} x \beta_{q}=x \alpha_{q}$ such that $x d_{i} \alpha_{q}=0$ and hence $d_{i} \alpha_{q}=0$.

The last statement follows directly by the formulas for the degeneracy maps.

By a $(p, q)$-shuffle $(\mu, \nu)$ we understand two ordered subsequences $\mu_{1}<$ $\cdots<\mu_{p}$ and $\nu_{1}<\cdots<\nu_{q}$ of the set $\{0,1, \ldots, p+q-1\}$ such that

$$
\left\{\mu_{1}, \ldots, \mu_{p}\right\} \sqcup\left\{\nu_{1}, \ldots, \nu_{q}\right\}=\{0,1, \ldots, p+q-1\} .
$$

Furthermore, we use the notation $s_{\mu}=s_{\mu_{p}} \ldots s_{\mu_{1}}$.

Lemma 2.4. Assume $(\mu, \nu)$ is a $(p, q)$-shuffle and $j \geq 1$. Then $s_{\mu}\left(y_{j}\right)=y_{\nu_{j}+1}$ and hence

$$
s_{\mu}\left(\omega_{q}\right)=\prod_{t \in\left\{\nu_{1}, \ldots, \nu_{q}\right\}} \mathbf{d} y_{t+1} \quad, \quad s_{\mu}\left(\left(\omega_{q}\right)_{j}\right)=\prod_{t \in\left\{\nu_{1}, \ldots, \widehat{\left.\nu_{j}, \ldots, \nu_{q}\right\}}\right.} \mathbf{d} y_{t+1} .
$$

Proof. We compute $s_{\mu}\left(y_{j}\right)$ by the formulas for the degeneracy maps. If $\mu_{1} \geq j$ then $\nu_{j}=j-1$ and $s_{\mu}\left(y_{j}\right)=y_{j}$. If $\mu_{2} \geq j+1$ and $\mu_{1}<j$ then $\nu_{j}=j$ and $s_{\mu}\left(y_{j}\right)=y_{j+1}$. In general, if $\mu_{r+1} \geq j+r$ and $\mu_{r}<j+r-1$ then $\nu_{j}=j+r-1$ and $s_{\mu}\left(y_{j}\right)=y_{j+r}$.

By results of Bousfield and Dwyer [D] we have that $H_{*}\left(T_{n}(x) ; \Omega\right)=$ $\pi_{*} \Omega\left(R_{\bullet}\right)$ is a graded $\mathbb{F}_{2}$-algebra equipped with homotopy operations

$$
\delta_{i}: H_{q}\left(T_{n}(x) ; \Omega\right) \rightarrow H_{q+i}\left(T_{n}(x) ; \Omega\right) \quad, \quad 2 \leq i \leq q .
$$

The following result gives a complete determination of this structure.

Theorem 2.5. Let $n$ be a positive integer. For $n$ odd, there is an isomorphism of algebras

$$
H_{*}\left(T_{n}(x) ; \Omega\right) \cong T_{n}(x) \otimes \Lambda(\mathbf{d} x) \otimes \Gamma[\omega]
$$


where $x, \mathbf{d} x \in H_{0}$ and $\gamma_{q}(\omega) \in H_{q}$ The homotopy operations are given by

$$
\delta_{i}\left(\gamma_{q}(\omega)\right)= \begin{cases}\left(\begin{array}{c}
2 q-1 \\
q
\end{array}\right) \gamma_{2 q}(\omega) & , i=q \\
0 & , i<q .\end{cases}
$$

The algebra generators are represented by cycles in the normalized chain complex $\left(N_{*} \Omega R, d_{0}\right)$ with $N_{q} \Omega R=\cap_{i>0} \operatorname{ker} d_{i}$ as follows: $x=[x], \mathbf{d} x=[\mathbf{d} x]$ and $\gamma_{i}(\omega)=\left[\omega_{i}\right]$.

For $n$ even, we have

$$
H_{*}\left(T_{n}(x) ; \Omega\right) \cong \mathbb{F}_{2} \oplus \mathbb{F}_{2}[x] /\left(x^{n}\right)<a_{q}, b_{q} \mid q \geq 0>
$$

where $x \in H_{0}$ and $a_{q}, b_{q} \in H_{q}$. The algebra structure is given by the relations

$$
a_{p} a_{q}=0, \quad b_{p} b_{q}=\left(\begin{array}{c}
p+q \\
p
\end{array}\right) x b_{p+q}, \quad a_{p} b_{q}=\left(\begin{array}{c}
p+q \\
p
\end{array}\right) x a_{p+q} .
$$

The homotopy operations are given by $\delta_{i}\left(a_{q}\right)=0$ and

$$
\delta_{i}\left(b_{q}\right)= \begin{cases}\left(\begin{array}{c}
2 q-1 \\
q
\end{array}\right) x b_{2 q} & , i=q \\
0 & , i<q .\end{cases}
$$

The generators are represented by cycles as follows: $x=[x], a_{q}=\left[\alpha_{q}\right]$, $b_{q}=\left[\beta_{q}\right]$.

Remark 2.6. The description in the theorem implies that $H_{0}\left(T_{n}(x) ; \Omega\right) \cong$ $\Omega\left(T_{n}(x)\right)$. Note also that

$$
\left(\begin{array}{c}
2 q-1 \\
q
\end{array}\right)= \begin{cases}1 & , q=2^{r}, r \geq 0 \\
0 & , \text { otherwise }\end{cases}
$$

Proof. We start by computing the derived functors additively. The algebra $T_{n}(x)$ is the pushout of the diagram $\mathbb{F}_{2} \leftarrow \mathbb{F}_{2}[y] \rightarrow \mathbb{F}_{2}[x]$ where $y \mapsto x^{n+1}$. Note that $\mathbb{F}_{2}[x]$ is free as a module over $\mathbb{F}_{2}[y]$. By BO1] Proposition 6.3 there is a Quillen spectral sequence as follows:

$$
E_{i, j}^{2}=\operatorname{Tor}_{i}^{H_{*}\left(\mathbb{F}_{2}[y] ; \Omega\right)}\left(\mathbb{F}_{2}, H_{*}\left(\mathbb{F}_{2}[x] ; \Omega\right)\right)_{j} \Rightarrow H_{i+j}\left(T_{n}(x) ; \Omega\right) .
$$

The algebras $\mathbb{F}_{2}[x]$ and $\mathbb{F}_{2}[y]$ are free objects in $\mathcal{A l g}$ so we find that $E_{i, j}^{2}=0$ for $j>0$ and

$$
H_{i}\left(T_{n}(x) ; \Omega\right) \cong E_{i, 0}^{2} \cong \operatorname{Tor}_{i}^{\Omega\left(\mathbb{F}_{2}[y]\right)}\left(\mathbb{F}_{2}, \Omega\left(\mathbb{F}_{2}[x]\right)\right)
$$


There is a resolution $\left(K_{*}, \partial\right)$ of $\mathbb{F}_{2}$ by free $\Omega\left(\mathbb{F}_{2}[y]\right)$-modules as follows:

$$
K_{*}=\Lambda(v) \otimes \Gamma[w] \otimes \Omega\left(\mathbb{F}_{2}[y]\right), \quad \partial v=y, \quad \partial \gamma_{i}(w)=\gamma_{i-1}(w) d y
$$

where $v \in K_{1}$ and $\gamma_{i}(w) \in K_{i}$. We tensor $K_{*}$ with $\Omega\left(\mathbb{F}_{2}[x]\right)$ over $\Omega\left(\mathbb{F}_{2}[y]\right)$ and get a complex $\left(C_{*}, \partial\right)$ with

$$
C_{*}=\Lambda(V) \otimes \Gamma[w] \otimes \Omega\left(\mathbb{F}_{2}[x]\right), \quad \partial v=x^{n+1}, \quad \partial \gamma_{i}(w)=(n+1) \gamma_{i-1}(w) x^{n} d x
$$

By computing the homology of $\left(C_{*}, \partial\right)$ we find that for $n$ even $H_{0}\left(T_{n}(x) ; \Omega\right)=$ $\Omega\left(T_{n}(x)\right)$ and

$$
H_{q}\left(T_{n}(x) ; \Omega\right)=\mathbb{F}_{2}[x] /\left(x^{n}\right)<d x \gamma_{q}(w), x \gamma_{q}(w)+d x v \gamma_{q-1}(w)>, \quad q>0 .
$$

For $n$ odd we find

$$
H_{*}\left(T_{n}(x) ; \Omega\right) \cong \Omega\left(T_{n}(x)\right) \otimes \Gamma[w] .
$$

We now show that there is an additive basis for $H_{q}\left(T_{n}(x) ; \Omega\right)$ which is represented by cycles in the normalized complex as stated. Lemma 2.3 shows that these representatives are indeed cycles. It suffices to show that the associated classes are linearly independent since, in each degree, the number of classes agree with the dimension in that degree.

We introduce two gradings on $\Omega R \bullet$ preserved by $d_{i}$. The wedge grading $w$ which equals the number of wedge factors and the polynomial grading $p$ with $p(x)=2, p(d x)=1, p\left(y_{i}\right)=2 n+2, p\left(d y_{i}\right)=2 n+1$ extended multiplicatively. Let $\Omega^{r, s}\left(R_{q}\right)$ denote the subspace of elements $\omega \in \Omega\left(R_{q}\right)$ with $w(\omega)=r$ and $p(\omega)=s$. We have defined a splitting of simplicial $\mathbb{F}_{2}$-vector spaces so it follows that

$$
H_{q}\left(T_{n}(x) ; \Omega\right) \cong \bigoplus_{r, s \geq 0} H_{q}\left(T_{n}(x) ; \Omega^{r, s}\right) .
$$

Assume that $n$ is odd. We must show that for fixed $q$ the elements

$$
x^{t}(\mathbf{d} x)^{\epsilon} \omega_{q}, \quad 0 \leq t \leq n, \epsilon \in\{0,1\}
$$

are linearly independent. The bidegree is given by $(q+\epsilon, 2 t+\epsilon+q(2 n+1))$. The elements have different bigradings and it suffices to check that they individually do not represent zero. Recall that the composite $N_{*}(V) \rightarrow$ $C_{*}(V) \rightarrow C_{*}(V) / D_{*}(V)$ is an isomorphism for any simplicial $\mathbb{F}_{2^{-}}$vector space $V_{\bullet}$ so we have that $N_{*} \Omega\left(R_{\bullet}\right) \cap D_{*} \Omega\left(R_{\bullet}\right)=0$.

We first check that $\left[x^{t} \mathbf{d} x \omega_{q}\right] \neq 0$. The chains which might hit the representative lie in $\Omega^{q+1,2 t+1+q(2 n+1)}\left(R_{q+1}\right)$ which is spanned by the elements $x^{t} \mathbf{d} x\left(\omega_{q+1}\right)_{j}$ for $1 \leq j \leq q+1$ together with $\omega_{q+1}$ if $t=n$. By Lemma 2.3 all 
these elements are degenerate except for $\omega_{q+1}$. It follows that the only non trivial normalized chain in this bigrading is $\omega_{q+1}$. But $d_{0}\left(\mathbf{d} y_{1}\right)=0$ such that $d_{0} \omega_{q+1}=0$.

We then check that $\left[x^{t} \omega_{q}\right] \neq 0$. The chains which might hit the representative lie in $\Omega^{q, 2 t+q(2 n+1)}\left(R_{q+1}\right)$ which is spanned by the elements $x^{t}\left(\omega_{q+1}\right)_{r}$, $x^{t+n} \mathbf{d} x\left(\omega_{q+1}\right)_{j, k}$ together with $x^{t-1} \mathbf{d} x y_{r}\left(\omega_{q+1}\right)_{j, k}$ if $t>0$ where $1 \leq r \leq q+1$, $1 \leq j<k \leq q+1$. By Lemma 2.3] all these elements are degenerate. So there are no normalized chains that can have $x^{t} \omega_{q}$ as boundary.

Assume that $n$ is even. We must show that the elements

$$
x^{t} \alpha_{q}, x^{t} \beta_{q}, \quad 1 \leq t \leq n-1
$$

are linearly independent. The bidegrees are

$$
\left\|x^{t} \alpha_{q}\right\|=(q+1,2 t+1+q(2 n+1)) \quad, \quad\left\|x^{t} \beta_{q}\right\|=(q, 2 t+2+q(2 n+1))
$$

so the elements have different bigradings and it suffices to check that they individually do not represent zero.

We first check that $\left[x^{t} \alpha_{q}\right] \neq 0$. The chains which might hit the representative lie in $\Omega^{q+1,2 t+1+q(2 n+1)}\left(R_{q+1}\right)$ which is spanned by the elements $x^{t+1} \mathbf{d} x\left(\omega_{q+1}\right)_{r}$ for $1 \leq r \leq q+1$ By Lemma 2.3 all these elements are degenerate.

We then check that $\left[x^{t} \beta_{q}\right] \neq 0$. The chains which might hit the representative lie in $\Omega^{q, 2 t+2+q(2 n+1)}\left(R_{q+1}\right)$ which is spanned by the elements

$$
x^{t+1}\left(\omega_{q+1}\right)_{r}, \quad x^{t+1+n} \mathbf{d} x\left(\omega_{q+1}\right)_{j, k}, \quad x^{t} \mathbf{d} x y_{r}\left(\omega_{q+1}\right)_{j, k},
$$

for $1 \leq r \leq q+1,1 \leq j<k \leq q+1$. By Lemma 2.3] all these elements are degenerate.

We now prove that the algebra structure and homotopy operations are as stated. The product comes from the chain map

$$
\rho: C_{*}\left(\Omega R_{\bullet}\right) \otimes C_{*}\left(\Omega R_{\bullet}\right) \stackrel{g}{\longrightarrow} C_{*}\left(\Omega R_{\bullet} \otimes \Omega R_{\bullet}\right) \stackrel{C_{*}(\mu)}{\longrightarrow} C_{*}\left(\Omega R_{\bullet}\right),
$$

where $g$ denotes the Eilenberg-MacLane map [ML] VIII.8. We use the results from $\mathrm{BO} 2$ to find the homotopy operations

$$
\delta_{i}: H_{q}\left(T_{n}(x) ; \Omega\right) \rightarrow H_{q+i}\left(T_{n}(x) ; \Omega\right), \quad 2 \leq i \leq q .
$$

For a cycle $z \in N_{q} \Omega\left(R_{\bullet}\right)$ we have $\delta_{i}([z])=\left[\delta_{i}(z)\right]$ where

$$
\delta_{i}(z)=\sum_{(\mu, \nu) \in V(i, q)} s_{\nu}(z) s_{\mu}(z)
$$


Assume that $n$ is odd. By the formulas for the shuffle map and Lemma 2.4 we find that

$$
\rho\left(\omega_{p} \otimes \omega_{q}\right)=\sum_{(\mu, \nu)} s_{\nu}\left(\omega_{p}\right) s_{\mu}\left(\omega_{q}\right)=\sum_{(\mu, \nu)} \omega_{p+q}=\left(\begin{array}{c}
p+q \\
p
\end{array}\right) \omega_{p+q}
$$

where the sums are over all $(p, q)$ shuffles. Thus the algebra structure is as stated.

The top homotopy operation is as stated since by Lemma 2.4 we have

$$
\delta_{q}\left(\omega_{q}\right)=\sum_{(\mu, \nu) \in V(q, q)} s_{\nu}\left(\omega_{q}\right) s_{\mu}\left(\omega_{q}\right)=\sum_{(\mu, \nu), \mu_{1}=0} \omega_{2 p}=\left(\begin{array}{c}
2 p-1 \\
p-1
\end{array}\right) \omega_{2 p} .
$$

For the other homotopy operations we note that when $(\mu, \nu) \in V(i, q)$ for $2 \leq i \leq q-1$ then both $s_{\nu}\left(\omega_{q}\right)$ and $s_{\mu}\left(\omega_{q}\right)$ contains a factor $\mathbf{d} y_{1}$ by Lemma 2.4 so the operations vanish since $\left(\mathbf{d} y_{1}\right)^{2}=0$.

Assume that $n$ is even. We have that $\rho\left(\alpha_{p} \otimes \alpha_{q}\right)=0$. So the first product formula holds. By the formulas defining $\beta_{p}$ and $\beta_{q}$ we find that

$$
\begin{aligned}
\rho\left(\beta_{p} \otimes \beta_{q}\right) & =x^{2} \rho\left(\omega_{p} \otimes \omega_{q}\right) \\
& +x \mathbf{d} x\left(\sum_{i=1}^{p} \rho\left(y_{i}\left(\omega_{p}\right)_{i} \otimes \omega_{q}\right)+\sum_{j=1}^{q} \rho\left(\omega_{p} \otimes y_{j}\left(\omega_{q}\right)_{j}\right)\right) .
\end{aligned}
$$

The first term equals $\left(\begin{array}{c}p+q \\ p\end{array}\right) x^{2} \omega_{p+q}$. By Lemma 2.4 we find that

$$
\rho\left(\omega_{p} \otimes y_{j}\left(\omega_{q}\right)_{j}\right)=\sum_{(\mu, \nu)} s_{\nu}\left(\omega_{p}\right) s_{\mu}\left(y_{j}\right) s_{\mu}\left(\left(\omega_{q}\right)_{j}\right)=\sum_{(\mu, \nu)} y_{\nu_{j}+1}\left(\omega_{p+q}\right)_{\nu_{j}+1}
$$

and by symmetry

$$
\rho\left(y_{i}\left(\omega_{p}\right)_{i} \otimes \omega_{q}\right)=\sum_{(\mu, \nu)} y_{\mu_{i}+1}\left(\omega_{p+q}\right)_{\mu_{i}+1}
$$

We insert these above and find that $\rho\left(\beta_{p} \otimes \beta_{q}\right)=\left(\begin{array}{c}p+q \\ p\end{array}\right) x \beta_{p+q}$. This gives the second formula for the product. The third follows by multiplication with $\mathbf{d} x$ on the chain level.

A similar argument shows that $\delta_{q}\left(\beta_{q}\right)=\left(\begin{array}{c}2 q-1 \\ q-1\end{array}\right) x \beta_{2 q}$. For $2 \leq i \leq q-1$ we get $\delta_{i}\left(\beta_{q}\right)=0$ since $\left(\mathbf{d} y_{1}\right)^{2}=0$. Finally, we have that $\delta_{k}\left(\alpha_{q}\right)=0$ for all $k$ since $(\mathbf{d} x)^{2}=0$. 


\section{$3 \quad$ Normalized chain complex manipulations}

This very technical section is needed in the discussion of the Steenrod algebra action in the next section.

Let $A$. be a simplicial ring. In a DGA it follows directly from the definition that the product of two cycles is a cycle, and that the product of a cycle with a boundary is a boundary. The Eilenberg-Zilber equivalence allows you to transfer this statement to simplicial rings. We will only use a rather special case.

Definition 3.1. Let $m(a, b)=s_{0}(a) s_{1}(b)-s_{0}(b) s_{1}(a) \in A_{n+1}$ and $q(a)=$ $s_{0}(a) s_{1}(a) \in A_{n+1}$ for $a, b \in A_{n}$.

When $n=1$ the map $m$ can be described as the shuffle map followed by the multiplication map $A_{1} \otimes A_{1} \stackrel{g}{\rightarrow} A_{2} \otimes A_{2} \stackrel{\mu}{\rightarrow} A_{2}$.

The bilinear map $m$ is skew-symmetric and $m(a, a)=0$. In case $A_{\bullet}$ is a simplicial $\mathbb{F}_{2}$-algebra we have that $q(a+b)=q(a)+q(b)+m(a, b)$ so the two constructions formally behave like a symmetric bilinear form and the quadratic form belonging to it.

We will show that $m$ preserves the normalized chain complex, and pass to well-defined operations on its homology. The situation for $q$ is similar, but slightly more complicated.

Lemma 3.2. Assume that $a, b \in N_{n}(A)$ and $c \in A_{n-1}$ (in particular we may take $c=1$ when $A$. is unital). Then

- $s_{1} s_{0}(c) m(a, b) \in N_{n+1}(A)$.

- If $d_{0}(a) c=0=d_{0}(b) c$, then $s_{1} s_{0}(c) m(a, b)$ is a cycle.

- If $d_{0}(a) c=0$ is a cycle and $b$ is a boundary, then $s_{1} s_{0}(c) m(a, b)$ is a boundary.

Proof. We compute the face maps on the element $z=s_{0} s_{0}(c) m(a, b)$.

$$
\begin{aligned}
& d_{0}(z)=s_{0}(c)\left(a s_{0} d_{0}(b)-s_{0} d_{0}(a) b\right)=a s_{0}\left(c d_{0}(b)\right)-s_{0}\left(c d_{0}(a)\right) b, \\
& d_{1}(z)=s_{0}(c)(a b-a b)=0, \\
& d_{2}(z)=s_{0} s_{0} d_{0}(c)\left[s_{0} d_{1}(a) b-a s_{0} d_{1}(b)\right]=0, \\
& d_{i}(z)=s_{0} s_{0} d_{i-2}(c)\left[s_{0} d_{i-1}(a) s_{1} d_{i-1}(b)-s_{1} d_{i-1}(a) s_{0} d_{i-1}(b)\right]=0 \text { for } i \geq 3 .
\end{aligned}
$$

The first two statements follows from this.

Now assume that $d_{0}(a) c=0$ and that $b=d_{0}(x)$ for some $x \in N_{n+1}(A)$. We define

$$
y=s_{2} s_{1} s_{0}(c)\left[s_{2} s_{1}(a) s_{0}(x)-s_{2} s_{0}(a) s_{1}(x)+s_{1} s_{0}(a) s_{2}(x)\right] \in A_{n+2} .
$$


Note that this element is derived from the shuffle map. We compute face maps:

$$
\begin{aligned}
d_{0}(y) & =s_{1} s_{0}(c)\left[s_{1} s_{0} d_{0}(a) x-s_{1}(a) s_{0} d_{0}(x)+s_{0}(a) s_{1} d_{0}(x)\right] \\
& =s_{1} s_{0}(c) s_{1} s_{0} d_{0}(a) x+s_{1} s_{0}(c)\left[s_{0}(a) s_{1}(b)-s_{1}(a) s_{0}(b)\right] \\
& =s_{1} s_{0}\left(c d_{0}(a)\right) x+s_{1} s_{0}(c) m(a, b)=s_{1} s_{0}(c) m(a, b), \\
d_{1}(y) & =s_{1} s_{0}(c)\left[s_{1}(a) x-s_{1}(a) x+s_{0}(a) s_{1} d_{1}(x)\right]=0, \\
d_{2}(y) & =s_{1} s_{0}(c)\left[s_{1}(a) s_{0} d_{1}(x)-s_{0}(a) x+s_{0}(a) x\right]=0, \\
d_{i}(y) & =s_{1} s_{0} d_{i-2} s_{0}(c)\left[s_{2} s_{1} d_{i-2}(a) s_{0} d_{i-1}(x)\right. \\
& \left.-s_{2} s_{0} d_{i-2}(a) s_{1} d_{i-1}(x)+s_{1} s_{0} d_{i-2}(a) s_{2} d_{i-1}(x)\right]=0 \text { for } i \geq 3 .
\end{aligned}
$$

Lemma 3.3. Assume that $a \in N_{n}(A), b \in N_{n+1}(A)$ and $c \in A_{n}$ (in particular we may take $c=1$ if $A_{\bullet}$ is unital).

- If $c a^{2}=0$, then $s_{0}(c) q(a) \in N_{n+1}(A)$.

- If in addition $\operatorname{cas}_{0} d_{0}(a)=0$ (in particular, if $a$ is a cycle), then $s_{0}(c) q(a)$ is a cycle.

- If $s_{0}(c) b^{2}=0$, then $s_{0}(c) q\left(d_{0}(b)\right)$ is a boundary.

Proof. Put $y=s_{0}(c) q(a)=s_{0}(c) s_{0}(a) s_{1}(a)$. The first two statements follows by the computation

$$
\begin{aligned}
& d_{0}(y)=\operatorname{cas}_{0} d_{0}(a) \\
& d_{1}(y)=c a^{2}=0 \\
& d_{2}(y)=s_{0} d_{1}(c) s_{0} d_{1}(a) a=0 \\
& d_{i}(y)=s_{0} d_{i-1}(c) s_{0} d_{i-1}(a) s_{1} d_{i-1}(a)=0 \text { if } i \geq 3 .
\end{aligned}
$$

We claim that if $s_{0}(c) b^{2}=0$, then $s_{1} s_{0}(c) s_{1}(b) s_{2}(b)$ is a chain with boundary $s_{0}(c) q\left(d_{0}(b)\right)$. The claim follows by the computation:

$$
d_{i}\left(s_{1} s_{0}(c) s_{1}(b) s_{2}(b)\right)= \begin{cases}s_{0}(c) s_{0} d_{0}(b) s_{1} d_{0}(b)=s_{0}(c) q\left(d_{0}(b)\right) & \text { if } i=0, \\ s_{0}(c) b s_{1} d_{1}(b)=s_{0}(c) b \cdot 0=0 & \text { if } i=1, \\ s_{0}(c) b^{2}=0 & \text { if } i=2, \\ s_{1} s_{0} d_{i-2}(c) s_{1} d_{i-1}(b) s_{2} d_{i-1}(b)=0 & \text { if } i \geq 3 .\end{cases}
$$




\section{The Steenrod algebra action}

The algebra $T_{n}(x)$ is sometimes an object in $\mathcal{K}$. For $n \geq 2$ this happens if and only if $|x|$ is a power of two. For $n=1$ it happens if and only if $|x| \geq 1$. There is at most one $\mathcal{A}$-action which makes $T_{n}(x)$ an object in $\mathcal{K}$.

For any $K \in \mathcal{K}$ there is an $\mathcal{A}$-action on $H_{*}(K ; \bar{\Omega})$. In this section we determine this action when $K=T_{n}(x) \in \mathcal{K}$.

We first consider the case where $|x|$ is a power of two. Define $T_{n, t}(x) \in K$ for $n \geq 1, t \geq 0$ by $T_{n, t}(x)=\mathbb{F}_{2}[x] /\left(x^{n+1}\right)$ with $m=|x|=2^{t}$. It is an object in $\mathcal{K}$ by the formula

$$
\mathrm{Sq}^{i}\left(x^{j}\right)=\left(\begin{array}{c}
m j \\
i
\end{array}\right) x^{j+j / m}
$$

Let $\Phi: \mathcal{K} \rightarrow \mathcal{K}$ denote the doubling functor with $\Phi(K)^{2 j}=K^{j}$ and $\Phi(K)^{2 j+1}=0$ for $K \in \mathcal{K}$. The operation $\mathrm{Sq}^{2 i}$ act on $\Phi(K)$ via the action of $\mathrm{Sq}^{i}$ on $K$. The operation $\mathrm{Sq}^{2 i+1}$ acts trivially on $\Phi(K)$. By BO1] Theorem 6.4 and Theorem 7.6 we have the following result:

Proposition 4.1. Let $A \in \mathcal{K}$ and assume that $A=\Phi^{t}(K)$ for some $t \geq 1$ and $K \in \mathcal{K}$. Then there is an isomorphism of $\mathcal{A}$-modules for all $q$ :

$$
H_{q}(A ; \bar{\Omega}) \cong \pi_{q} \Omega \Phi^{t}\left(B_{\bullet}\right)
$$

where $B_{\bullet} \in$ sK $\mathcal{K}$ is an almost free resolution of $K$.

Note that $T_{n, t}(x)=\Phi^{t} T_{n, 0}(x)$ where the $\mathcal{A}$-action on $T_{n, 0}(x)$ is given by

$$
\mathrm{Sq}^{j} x^{i}=\left(\begin{array}{l}
i \\
j
\end{array}\right) x^{i+j}
$$

Let $R_{\bullet}^{\mathcal{K}} \in s \mathcal{K}$ denote a simplicial resolution of $T_{n, 0}(x) \in \mathcal{K}$. There is a simplicial algebra map $R_{\bullet} \rightarrow R_{\bullet}^{\mathcal{K}}$ which induces a homotopy equivalence. We will abuse the notation, and denote the images in $R_{q}^{\mathcal{K}}$ of the classes $x, y_{1}, \ldots, y_{q} \in R_{q}$ by the same names.

By the formulas for the boundary maps and (11) we see that the element $\mathrm{Sq}^{i}\left(y_{1}\right)+\left(\begin{array}{c}n+1 \\ i\end{array}\right) x^{i} y_{1} \in R_{1}^{\mathcal{K}}$ is a cycle for $1 \leq i \leq n+1$. Since $R_{\bullet}^{\mathcal{K}}$ is a resolution of a discrete ring, $\pi_{k}\left(R_{\bullet}\right)=0$ for $k>0$ and we can find elements $z^{(i)} \in R_{2}^{\mathcal{K}}$ for $1 \leq i \leq n+1$ such that

$$
d_{0}\left(z^{(i)}\right)=\mathrm{Sq}^{i}\left(y_{1}\right)+\left(\begin{array}{c}
n+1 \\
i
\end{array}\right) x^{i} y_{1}, \quad d_{1}\left(z^{(i)}\right)=d_{2}\left(z^{(i)}\right)=0 .
$$

As we shall see below this information on $R_{\bullet}^{\mathcal{K}}$ turns out to be sufficient for the computation of the $\mathcal{A}$-action on $H_{*}\left(T_{n, t}(x) ; \bar{\Omega}\right)$. 
Theorem 4.2. Let $n \geq 1, t \geq 1$ and assume that $n$ is odd. Then the $\mathcal{A}$-action on

$$
H_{*}\left(T_{n, t}(x) ; \bar{\Omega}\right) \cong T_{n, t}(x) \otimes \Lambda(\mathbf{d} x) \otimes \Gamma[\omega]
$$

is given by the following formulas where $i, q \geq 0$ :

$$
\begin{aligned}
& \mathrm{Sq}^{2^{t_{i}}}\left(\gamma_{q}(\omega)=\left(\begin{array}{c}
q(n+1) \\
i
\end{array}\right) x^{i} \gamma_{q}(\omega)\right. \\
& \mathrm{Sq}^{2^{t_{i}}}\left(x^{j}\right)=\left(\begin{array}{c}
j \\
i
\end{array}\right) x^{i+j} \\
& \mathrm{Sq}^{2^{t} i}(\mathbf{d} x)= \begin{cases}\mathbf{d} x & , i=0 \\
0 & , i>0 .\end{cases}
\end{aligned}
$$

If $k \neq 0$ mod $2^{t}$ then $\mathrm{Sq}^{k}(z)=0$ for all $z \in H_{*}\left(T_{n, t}(x) ; \bar{\Omega}\right)$.

Proof. By Proposition 4.1 it suffices to prove the theorem for $t=1$. We use the simplicial object $\Phi\left(R_{\bullet}^{\mathcal{K}}\right)$ for our computations. We already know that (3) and (4) hold. Formula (2) holds for $q=0$ and also for $q=1$ since

$$
d_{0}\left(\mathbf{d} z^{(j)}\right)=\mathrm{Sq}^{2 j} \mathbf{d} y_{1}+\left(\begin{array}{c}
n+1 \\
j
\end{array}\right) x^{j} \mathbf{d} y_{1}, \quad 1 \leq j \leq n+1 .
$$

We now prove that (2) also holds for $q=2$. Let $v_{j}$ and $w_{j}$ denote the following elements for $0 \leq j \leq n+1: v_{j}=\mathrm{Sq}^{2 j} \mathbf{d} y_{1}$ and $w_{j}=\left(\begin{array}{c}n+1 \\ j\end{array}\right) x^{j} \mathbf{d}\left(y_{1}\right)$. Recall the constructions $m$ and $q$ from section 3

Claim 1: $m\left(v_{j}, v_{k}\right)$ is a boundary for $j \neq k$.

We have $d_{1} v_{j}=d_{1} w_{j}=0$ since $d_{1} y_{1}=0$. Furthermore, $d_{0} v_{j}=0$ and $d_{0} w_{j}=0$ since $n$ is odd. Thus $v_{j}$ and $w_{j}$ are cycles and $v_{j}+w_{j}$ is a boundary (actually 0 for $j=0$ ). Note that

$$
m\left(v_{j}, v_{k}\right)=m\left(v_{j}+w_{j}, v_{k}\right)+m\left(w_{j}, v_{k}+w_{k}\right)+m\left(w_{j}, w_{k}\right) .
$$

The first two terms of the right hand side are boundaries by Lemma 3.2. We now show that the last term $m\left(w_{j}, w_{k}\right)$ vanish and Claim 1 follows.

$$
\begin{aligned}
m\left(w_{j}, w_{k}\right) & =s_{0}\left(w_{j}\right) s_{1}\left(w_{k}\right)+s_{0}\left(w_{k}\right) s_{1}\left(w_{j}\right) \\
& =\left(\begin{array}{c}
n+1 \\
j
\end{array}\right)\left(\begin{array}{c}
n+1 \\
k
\end{array}\right)\left(\mathbf{d}\left(x^{j} y_{2}\right) \mathbf{d}\left(x^{k} y_{1}\right)+\mathbf{d}\left(x^{j} y_{1}\right) \mathbf{d}\left(x^{k} y_{2}\right)\right)
\end{aligned}
$$

so $m\left(w_{j}, w_{k}\right)=0$ unless $j$ and $k$ are both even. If they are we get

$$
m\left(w_{j}, w_{k}\right)=\left(\begin{array}{c}
n+1 \\
i
\end{array}\right)\left(\begin{array}{c}
n+1 \\
j
\end{array}\right) x^{j+k}\left(\mathbf{d} y_{2} \mathbf{d} y_{1}+\mathbf{d} y_{1} \mathbf{d} y_{2}\right)=0 .
$$


Claim 2: $q\left(v_{j}\right)$ is a cycle for $j>0$ which represents the same homology class as $\left(\begin{array}{c}n+1 \\ j\end{array}\right) x^{2 j} \mathbf{d} y_{1} \mathbf{d} y_{2}$.

By Lemma 3.3 we see that $q\left(v_{j}\right)$ is a cycle. Note that $q\left(v_{j}+w_{j}\right)=$ $q\left(v_{j}\right)+q\left(w_{j}\right)+m\left(v_{j}, w_{j}\right)$. By Lemma 3.3 we have that $q\left(v_{j}+w_{j}\right)$ is a boundary. Furthermore, $m\left(v_{j}, w_{j}\right)$ is a boundary since $m\left(v_{j}, w_{j}\right)=m\left(v_{j}+w_{j}, w_{j}\right)+$ $m\left(w_{j}, w_{j}\right)$ and $m\left(v_{j}+w_{j}, w_{j}\right)$ is a boundary by Lemma 3.2 and $m\left(w_{j}, w_{j}\right)=0$. Thus $q\left(v_{j}\right)+q\left(w_{j}\right)$ is a boundary. But

$$
\begin{aligned}
q\left(w_{j}\right) & =s_{0}\left(w_{j}\right) s_{1}\left(w_{j}\right) \\
& =\left(\begin{array}{c}
n+1 \\
j
\end{array}\right)\left(\begin{array}{c}
n+1 \\
j
\end{array}\right) \mathbf{d}\left(x^{j} y_{1}\right) \mathbf{d}\left(x^{j} y_{2}\right)=\left(\begin{array}{c}
n+1 \\
j
\end{array}\right) x^{2 j} \mathbf{d} y_{1} \mathbf{d} y_{2}
\end{aligned}
$$

so Claim 2 follows.

We can now prove formula (2) for $q=2$. By the Cartan formula we have

$$
\begin{aligned}
\mathrm{Sq}^{2 i}\left(\omega_{2}\right)= & \operatorname{Sq}^{2 i}\left(\mathbf{d} y_{1} \mathbf{d} y_{2}\right) \\
= & \sum_{j+k=i} \operatorname{Sq}^{2 j}\left(\mathbf{d} y_{1}\right) \operatorname{Sq}^{2 k}\left(\mathbf{d} y_{2}\right) \\
= & \sum_{j+k=i, j<k}\left(\operatorname{Sq}^{2 j}\left(\mathbf{d} y_{1}\right) \operatorname{Sq}^{2 k}\left(\mathbf{d} y_{2}\right)+\operatorname{Sq}^{2 j}\left(\mathbf{d} y_{2}\right) \operatorname{Sq}^{2 k}\left(\mathbf{d} y_{1}\right)\right) \\
& +\mathrm{Sq}^{i / 2}\left(\mathbf{d} y_{1}\right) \mathrm{Sq}^{i / 2}\left(\mathbf{d} y_{2}\right)
\end{aligned}
$$

where as usual we put $\mathrm{Sq}^{i / 2}=0$ when $i$ is odd. We can rewrite this as

$$
\mathrm{Sq}^{2 i}\left(\mathbf{d} y_{1} \mathbf{d} y_{2}\right)=\sum_{j+k=i, j<k} m\left(v_{j}, v_{k}\right)+q\left(v_{i / 2}\right) .
$$

Now by Claim 1 and Claim 2 see that $\operatorname{Sq}^{2 i}\left(\mathbf{d} y_{1} \mathbf{d} y_{2}\right)$ represents the same homology class as $\left(\begin{array}{c}n+1 \\ i / 2\end{array}\right) x^{i} \mathbf{d} y_{1} \mathbf{d} y_{2}=\left(\begin{array}{c}2(n+1) \\ i\end{array}\right) x^{i} \omega_{2}$ for $i$ even and zero for $i$ odd. Since $\left(\begin{array}{c}2(n+1) \\ i\end{array}\right)=0$ for $i$ odd, the result follows.

By the formulas for the product structure and the homotopy operations we can now prove (2) in general. We start by proving (2) for $q=2^{r}$ with $r \geq 1$ by induction on $r$. The induction start is the formula for $q=2$, which we just proved. Assume that the formula holds for some $q=2^{r}$. Put $\gamma_{k}=\gamma_{k}(\omega)$ for all $k$. By Theorem 2.5 we have that $\gamma_{2 q}=\delta_{q}\left(\gamma_{q}\right)$. So we can use the general relations among homotopy operations and Steenrod operations $[\mathrm{G}$ :

$$
\begin{aligned}
\mathrm{Sq}^{2 i} \gamma_{2 q} & =\mathrm{Sq}^{2 i}\left(\delta_{q} \gamma_{q}\right)= \\
& =\delta_{q}\left(\mathrm{Sq}^{i} \gamma_{q}\right)+\sum_{k<i} \operatorname{Sq}^{k}\left(\gamma_{q}\right) \operatorname{Sq}^{i-k}\left(\gamma_{q}\right)
\end{aligned}
$$


By induction and by the formula for the product, the last term vanish. The first term vanish if $i$ is odd. If $i$ is even we get

$$
\mathrm{Sq}^{2 i} \gamma_{2 q}=\left(\begin{array}{c}
q(n+1) \\
i / 2
\end{array}\right) \delta_{q}\left(x^{i / 2} \gamma_{q}\right)=\left(\begin{array}{c}
q(n+1) \\
i / 2
\end{array}\right) x^{i} \gamma_{2 q}
$$

This proves (2) when $q$ is a power of 2 .

Finally, we use the product structure to prove (2) for all remaining $q$. Assume that it holds for all $q<2^{r}$ and consider a $p$ with $2^{r}<p<2^{r+1}$. We have $p=2^{r}+s$ where $0<s<2^{r}$. Thus $\gamma_{p}=\gamma_{2^{r}} \gamma_{s}$ and

$$
\begin{aligned}
\mathrm{Sq}^{2 i}\left(\gamma_{p}\right) & =\sum_{j=0}^{i} \operatorname{Sq}^{2 i}\left(\gamma_{2^{r}}\right) \mathrm{Sq}^{2(i-j)}\left(\gamma_{s}\right) \\
& =\sum_{j=0}^{i}\left(\begin{array}{c}
2^{r}(n+1) \\
j
\end{array}\right)\left(\begin{array}{c}
s(n+1) \\
i-j
\end{array}\right) x^{i} \gamma_{p}=\left(\begin{array}{c}
p(n+1) \\
i
\end{array}\right) x^{i} \gamma_{p}
\end{aligned}
$$

where we used the combinatorial interpretations of binomial coefficients to see the last equality. By induction on $r$ we are done.

Theorem 4.3. Let $n \geq 1, t \geq 1$ and assume that $n$ is even. Then the $\mathcal{A}$-action on $H_{*}\left(T_{n, t}(x) ; \bar{\Omega}\right)$ is given by the following formulas where $i, q \geq 0$ :

$$
\begin{aligned}
& \operatorname{Sq}^{2^{t} i}\left(a_{q}\right)=\left(\begin{array}{c}
q(n+1) \\
i
\end{array}\right) x^{i} a_{q} \\
& \operatorname{Sq}^{2^{t} i}\left(b_{q}\right)=\left(\begin{array}{c}
q(n+1)+1 \\
i
\end{array}\right) x^{i} b_{q} \\
& \operatorname{Sq}^{2^{t} i}\left(x^{j}\right)=\left(\begin{array}{l}
j \\
i
\end{array}\right) x^{i+j}
\end{aligned}
$$

If $k \neq 0 \bmod 2^{t}$ then $\operatorname{Sq}^{k}(z)=0$ for all $z \in H_{*}\left(T_{n, t}(x) ; \bar{\Omega}\right)$.

Proof. It is enough to prove the theorem for $t=1$. The proof for the case $n=2$ is given in Lemma 4.4. Assume that $n \geq 4$. We know that (7) holds. Equation (5) and (6) are OK for $q=0$ by definition.

For $q=1$ we use the elements $z^{(i)}, 1 \leq i \leq n$. Since

$$
d_{0}\left(\mathbf{d} x \mathbf{d} z^{(i)}\right)=\mathbf{d} x \mathrm{Sq}^{2 i} \mathbf{d} y_{1}+\left(\begin{array}{c}
n+1 \\
i
\end{array}\right) x^{i} \mathbf{d} x \mathbf{d} y_{1}
$$

we see that (5) holds for $q=1$. Equation (6) holds by the following (where we take $\left.z^{(0)}=0\right)$ : 


$$
\begin{aligned}
& d_{0}\left(x^{2} \mathbf{d} z^{(i-1)}+x \mathbf{d} z^{(i)}+z^{(i)} \mathbf{d} x\right)= \\
& x^{2} \operatorname{Sq}^{2 i-2}\left(\mathbf{d} y_{1}\right)+\left(\begin{array}{c}
n+1 \\
i-1
\end{array}\right)\left((i-1) x^{i} \mathbf{d} x y_{1}+x^{i+1} \mathbf{d} y_{1}\right) \\
+ & x \operatorname{Sq}^{2 i}\left(\mathbf{d} y_{1}\right)+\left(\begin{array}{c}
n+1 \\
i
\end{array}\right)\left(i x^{i} \mathbf{d} x y_{1}+x^{i+1} \mathbf{d} y_{1}\right) \\
+ & \operatorname{Sq}^{2 i}\left(y_{1}\right) \mathbf{d} x+\left(\begin{array}{c}
n+1 \\
i
\end{array}\right) x^{i} y_{1} \mathbf{d} x= \\
& \operatorname{Sq}^{2 i}\left(x \mathbf{d} y_{1}+y_{1} \mathbf{d} x\right)+\left(\begin{array}{c}
n+2 \\
i
\end{array}\right) x^{i}\left(x \mathbf{d} y_{1}+y_{1} \mathbf{d} x\right) .
\end{aligned}
$$

Note that the assumption, $n$ even, is used for the last equality.

For $q=2$ we have $\alpha_{2}=\mathbf{d} x \mathbf{d} y_{1}$. By the Cartan formula we find that

$$
\mathrm{Sq}^{2 i}\left(\alpha_{2}\right)=\mathbf{d} x \sum_{j+k=i, j<k} m\left(\mathrm{Sq}^{2 j} \mathbf{d} y_{1}, \mathrm{Sq}^{2 k} \mathbf{d} y_{1}\right)+\mathbf{d} x q\left(\mathrm{Sq}^{i} \mathbf{d} y_{1}\right) .
$$

Define $v_{j}=\mathrm{Sq}^{2 j} \mathbf{d} y_{1}$ and $w_{j}=\left(\begin{array}{c}n+1 \\ j\end{array}\right) x^{j} \mathbf{d} y_{1}$. Formula (15) for $q=2$ now follows by Claim 1 and Claim 2 below.

Claim 1: $\mathbf{d} x m\left(v_{j}, v_{k}\right)$ is a boundary when $j \neq k$.

We have that $v_{j}, w_{j} \in N_{1} \Omega\left(R_{\bullet}\right)$. Furthermore, $\mathbf{d} x v_{j}$ and $\mathbf{d} x w_{j}$ are cycles and $v_{j}+w_{j}$ is a boundary. Note that

$$
\mathbf{d} x m\left(v_{j}, v_{k}\right)=\mathbf{d} x m\left(v_{j}+w_{j}, v_{k}\right)+\mathbf{d} m\left(w_{j}, v_{k}+w_{k}\right)+\mathbf{d} x m\left(w_{j}, w_{k}\right) .
$$

The first two terms are boundaries by Lemma 3.2 and the last term equals zero by direct computation. Thus Claim 1 holds.

Claim 2: For $i>0$ the element $\mathbf{d} x q\left(\mathrm{Sq}^{2 i} y_{1}\right)$ is a cycle representing the same homology class as $\left(\begin{array}{c}n+1 \\ i\end{array}\right) x^{i} \alpha_{2}$.

We have the following equation:

$$
\mathbf{d} x q\left(v_{i}+w_{i}\right)=\mathbf{d} x q\left(v_{i}\right)+\mathbf{d} x q\left(w_{i}\right)+\mathbf{d} x m\left(v_{i}, w_{i}\right) .
$$

Since $\left(v_{i}+w_{i}\right)^{2}=v_{i}^{2}+w_{i}^{2}=0$ the left hand side is a boundary by Lemma 3.3. The last term on the right hand side is a boundary since $m\left(v_{i}, v_{i}\right)=0$ and

$$
\mathbf{d} x m\left(v_{i}, w_{i}\right)=\mathbf{d} x m\left(v_{i}, v_{i}\right)+\mathbf{d} x m\left(v_{i}, v_{i}+w_{i}\right) .
$$

Thus $\mathbf{d} x q\left(v_{i}\right)$ and $\mathbf{d} x q\left(w_{i}\right)$ represent the same homology class. But $\mathbf{d} x q\left(w_{i}\right)=$ $\left(\begin{array}{c}n+1 \\ i\end{array}\right) x^{i} \alpha_{2}$ so Claim 2 follows.

We now use the homotopy operations and the product structure to prove the remaining part of the theorem. Note that $\mathrm{Sq}^{2 i} b_{q}=C_{q, i} x^{i} b_{q}$ and $\mathrm{Sq}^{2 i} a_{q}=$ 
$D_{q, i} x^{i} a_{q}$ for some constants $C_{q, i}$ and $D_{q, i}$ in $\mathbb{F}_{2}$. By the following argument it suffices to show that the constants are as stated for $1 \leq i \leq n-2$. For $i=0$ the constants are 1 and for $i=n-1$ they can can be found from the $i=n-2$ constants via the relation $\mathrm{Sq}^{2} \mathrm{Sq}^{2(n-2)}=\mathrm{Sq}^{2(n-1)}$ which is the "double" of $\mathrm{Sq}^{1} \mathrm{Sq}^{n-2}=\mathrm{Sq}^{n-1}$. For $i \geq n$ there is nothing to show since $x^{n}=0$.

By the product relation $x a_{2}=\mathbf{d} x b_{2}$ we see that

$$
\begin{aligned}
& C_{2, i} x^{i+1} a_{2}=C_{2, i} x^{i} \mathbf{d} x b_{2}=\mathrm{Sq}^{2 i}\left(\mathbf{d} x b_{2}\right)=\mathrm{Sq}^{2 i}\left(x a_{2}\right)=x^{2} \mathrm{Sq}^{2 i-2} a_{2}+x \mathrm{Sq}^{2 i} a_{2} \\
& =\left(\begin{array}{c}
2(n+1) \\
i-1
\end{array}\right) x^{i+1} a_{2}+\left(\begin{array}{c}
2(n+1) \\
i
\end{array}\right) x^{i+1} a_{2}=\left(\begin{array}{c}
2(n+1)+1 \\
i
\end{array}\right) x^{i+1} a_{2} .
\end{aligned}
$$

Thus formula (66) holds for $q=2$. So we know that (5) and (6) holds for $q=0,1,2$.

We now prove that (6) holds when $q=2^{r}, r \geq 1$ by induction on $r$. The initial case $r=2$ holds by the above. Assume that we have the formula for some $r \geq 2$. By the general relations [G] we find that

$$
\mathrm{Sq}^{2 i}\left(\delta_{q}\left(b_{q}\right)\right)=\delta_{q}\left(\mathrm{Sq}^{i} b_{q}\right)+\sum_{t<i} \operatorname{Sq}^{t}\left(b_{q}\right) \mathrm{Sq}^{i-t}\left(b_{q}\right) .
$$

The last sum vanish since $b_{q}^{2}=0$. The first term vanish when $i$ is odd. When $i$ is even we use the induction hypothesis:

$$
\delta_{q}\left(\mathrm{Sq}^{i} b_{q}\right)=\left(\begin{array}{c}
q(n+1)+1 \\
i / 2
\end{array}\right) \delta_{q}\left(x^{i / 2} b_{q}\right)=\left(\begin{array}{c}
q(n+1)+1 \\
i / 2
\end{array}\right) x^{i+1} b_{2 q} .
$$

Thus the following formula holds for all $i$ :

$$
\mathrm{Sq}^{2 i}\left(\delta_{q}\left(b_{q}\right)\right)=\left(\begin{array}{c}
2 q(n+1)+2 \\
i
\end{array}\right) x^{i+1} b_{2 q} .
$$

But $\delta_{q}\left(b_{q}\right)=x b_{2 q}$ so this equals $x \mathrm{Sq}^{2 i}\left(b_{2 q}\right)+x^{2} \mathrm{Sq}^{2 i-2}\left(b_{2 q}\right)$ and we have

$$
C_{2 q, i-1}+C_{2 q, i}=\left(\begin{array}{c}
2 q(n+1)+2 \\
i
\end{array}\right) \text {. }
$$

Since $C_{0}=1$ we see that (6) holds for $q=2^{r+1}$.

We now know that (6) holds when $q$ is a power of 2 . We use the product structure to prove (6) for the remaining values of $q$. Assume that the formula holds for all $q<2^{r}$ and consider a $p$ with $2^{r}<p<2^{r+1}$. Then $p=2^{r}+s$ where $0<s<2^{r}$. We have

$$
\begin{aligned}
& \left(C_{p, i}+C_{p, i-1}\right) x^{i+1} b_{p}=x \mathrm{Sq}^{2 i} b_{p}+x^{2} \mathrm{Sq}^{2 i-2} b_{p}=\mathrm{Sq}^{2 i}\left(x b_{p}\right)=\mathrm{Sq}^{2 i}\left(b_{2^{r}} b_{s}\right) \\
& =\sum_{j=0}^{i} \operatorname{Sq}^{2 j}\left(b_{2^{r}}\right) \mathrm{Sq}^{2(i-j)}\left(b_{s}\right)=\sum_{j=0}^{i}\left(\begin{array}{c}
2^{r}(n+1)+1 \\
j
\end{array}\right)\left(\begin{array}{c}
s(n+1)+1 \\
i-j
\end{array}\right) x^{i} b_{2^{r}} b_{s} .
\end{aligned}
$$


By computing the sum of the binomial coefficients we find that

$$
D_{p, i}+D_{p, i-1}=\left(\begin{array}{c}
p(n+1)+2 \\
i
\end{array}\right)
$$

and since $C_{p, 0}=1$ the result follows.

Finally, we finish the proof of (15):

$$
\begin{aligned}
& \left(D_{q, i}+D_{q, i-1}\right) x^{i+1} a_{q}=x \mathrm{Sq}^{2 i} a_{q}+x^{2} \mathrm{Sq}^{2 i-2} a_{q}=\mathrm{Sq}^{2 i}\left(x a_{q}\right)=\operatorname{Sq}^{2 i}\left(\mathbf{d} x b_{q}\right) \\
& =\mathbf{d} x \mathrm{Sq}^{2 i} b_{q}=\left(\begin{array}{c}
q(n+1)+1 \\
i
\end{array}\right) \mathbf{d} x x^{i} b_{q}=\left(\begin{array}{c}
q(n+1)+1 \\
i
\end{array}\right) x^{i+1} a_{q} .
\end{aligned}
$$

The result follows since $D_{q, 0}=1$.

The homotopy operations and the product structure does not determine the Steenrod algebra action on $H_{*}\left(T_{n, t}(x) ; \bar{\Omega}\right)$ when $n=2$. We prove the formulas for the action on the chain level instead.

Let $R_{\bullet}^{\mathcal{K}} \in s \mathcal{K}$ be a simplicial resolution of $T_{2,0}(x)$. As above we have elements $x, y_{1}, \ldots, y_{q} \in R_{q}^{\mathcal{K}}$ for $q \geq 0$ and $z^{(1)} \in R_{2}^{\mathcal{K}}$.

Lemma 4.4. Put $S_{\bullet}=\Omega \Phi R_{\bullet}^{\mathcal{K}}$ and let $D$ denote the shuffle map and $\mu$ the product map as follows:

$$
S_{2} \otimes S_{q-2} \stackrel{D}{\longrightarrow} S_{q} \otimes S_{q} \stackrel{\mu}{\longrightarrow} S_{q}
$$

Define elements $A_{q}, B_{q} \in S_{2} \otimes S_{q-2}$ for $q \geq 2$ by

$$
A_{q}=\mathbf{d} x \mathbf{d} z^{(1)} \otimes \omega_{q-2} \quad, \quad B_{q}=\mathbf{d}\left(x z^{(1)}\right) \otimes \omega_{q-2}+\sum_{j=1}^{q-2} \mathbf{d} x \mathbf{d} z^{(1)} \otimes y_{j}\left(\omega_{q-2}\right)_{j} .
$$

Then one has that $\mu D\left(A_{q}\right), \mu D\left(B_{q}\right) \in N_{q}(S)$ and that

$$
\begin{aligned}
& d_{0} \mu D\left(A_{q}\right)=\mathrm{Sq}^{2} \alpha_{q-1}+(q-1) x \alpha_{q-1}, \\
& d_{0} \mu D\left(B_{q}\right)=\mathrm{Sq}^{2} \beta_{q-1}+q x \beta_{q-1} .
\end{aligned}
$$

Proof. By Proposition [7.1 it follows that $d_{i} \mu D\left(A_{q}\right)=0$ for $i>0$ and that

$$
\begin{aligned}
d_{0} \mu D\left(A_{q}\right) & =\mu D\left(d_{0} \otimes i d+i d \otimes d_{0}\right)\left(A_{q}\right) \\
& =\mu D\left(\mathbf{d} x \mathbf{d}\left(\operatorname{Sq}^{2}\left(y_{1}\right)+x y_{1}\right) \otimes \omega_{q-2}\right)+\mu D\left(\mathbf{d} x \mathbf{d} z^{(1)} \otimes x^{2} \mathbf{d} x \omega_{q-3}\right)
\end{aligned}
$$


Here the last term vanish since $(\mathbf{d} x)^{2}=0$. So by the formula for the shuffle map we find

$$
\begin{aligned}
d_{0} \mu D\left(A_{q}\right) & =\sum_{i=0}^{q-2} s_{q-2} \ldots \widehat{s_{i}} \ldots s_{0}\left(\mathbf{d} x \mathbf{d}\left(\mathrm{Sq}^{2}\left(y_{1}\right)+x y_{1}\right)\right) s_{i}\left(\omega_{q-2}\right) \\
& =\sum_{j=1}^{q-1} \mathbf{d} x\left(S q^{2}\left(\mathbf{d} y_{j}\right)+x \mathbf{d} y_{j}\right)\left(\omega_{q-1}\right)_{j} \\
& =\sum_{j=1}^{q-1} \mathbf{d} x \operatorname{Sq}{ }^{2}\left(\mathbf{d} y_{j}\right)\left(\omega_{q-1}\right)_{j}+\sum_{j=1}^{q-1} x \mathbf{d} x \omega_{q-1} \\
& =\mathrm{Sq}^{2} \alpha_{q-1}+(q-1) x \alpha_{q-1} .
\end{aligned}
$$

We now check that $d_{i} \mu D\left(B_{q}\right)=0$ for $i>0$. Since $d_{t}\left(z^{(1)}\right)=d_{t}\left(\omega_{q-2}\right)=0$ for $t>0$ we get the following by Proposition 7.1.

$$
d_{i} \mu D\left(B_{q}\right)=\sum_{j=1}^{q-2} \sum_{t=1}^{i} \mu G_{t}\left(\mathbf{d} x \mathbf{d} z^{(1)} \otimes d_{t}\left(y_{j}\left(\omega_{q-2}\right)_{j}\right)\right) .
$$

When $1 \leq t \leq q-3$ one has that $d_{t}\left(y_{j}\left(\omega_{q-2}\right)_{j}\right)=y_{j} \omega_{q-3}$ for $j=t, t+1$ and zero otherwise. When $t=q-2$ we always get zero. So $d_{i} \mu D\left(B_{q}\right)=0$ for $i>0$ as stated.

Finally, we show that $d_{0} \mu D\left(B_{q}\right)=\mathrm{Sq}^{2} \beta_{q-1}+q x \beta_{q-1}$. The right hand side gives the following:

$$
\begin{aligned}
& \mathrm{Sq}^{2} \beta_{q-1}+q x \beta_{q-1}= \\
& x \mathrm{Sq}^{2}\left(\omega_{q-1}\right)+x^{2} \omega_{q-1}+\mathbf{d} x \sum_{j=1}^{q-1} \mathrm{Sq}^{2}\left(y_{j}\right)\left(\omega_{q-1}\right)_{j}+\mathbf{d} x \sum_{j=1}^{q-1} y_{j} \mathrm{Sq}^{2}\left(\left(\omega_{q-1}\right)_{j}\right) \\
& +q x^{2} \omega_{q-1}+q x \mathbf{d} x \sum_{j=1}^{q-1} y_{j}\left(\omega_{q-1}\right)_{j}= \\
& x \mathrm{Sq}^{2}\left(\omega_{q-1}\right)+(q+1) x^{2} \omega_{q-1}+q x \mathbf{d} x \sum_{j=1}^{q-1} y_{j}\left(\omega_{q-1}\right)_{j}+\mathbf{d} x \sum_{j=1}^{q-1} \mathrm{Sq}^{2}\left(y_{j}\right)\left(\omega_{q-1}\right)_{j} \\
& +\mathbf{d} x \sum_{j=1}^{q-1} y_{j}\left(\sum_{k=1}^{j-1} \mathrm{Sq}^{2}\left(\mathbf{d} y_{k}\right)\left(\omega_{q-1}\right)_{k, j}+\sum_{k=j+1}^{q-1} \mathrm{Sq}^{2}\left(\mathbf{d} y_{k}\right)\left(\omega_{q-1}\right)_{j, k}\right)
\end{aligned}
$$

For the left hand side, we use that $d_{0} \mu D=\mu D\left(d_{0} \otimes i d+i d \otimes d_{0}\right)$ by Proposition [7.1. Since $(\mathbf{d} x)^{2}=0$ we find that

$$
\mu D\left(i d \otimes d_{0}\right)\left(B_{q}\right)=\mu D\left(\mathbf{d}\left(x z^{(1)}\right) \otimes x^{2} \mathbf{d} x \omega_{q-3}+\mathbf{d} x \mathbf{d} z^{(1)} \otimes x^{3} \omega_{q-3}\right)=0
$$


and hence

$$
\begin{aligned}
& d_{0} \mu D\left(B_{q}\right)=\mu D\left(d_{0} \otimes i d\right)\left(B_{q}\right) \\
& =\mu D\left(\mathbf{d}\left(x d_{0} z^{(1)}\right) \otimes \omega_{q-2}\right)+\mathbf{d} x \sum_{j=1}^{q-2} \mu D\left(\mathbf{d}\left(d_{0} z^{(1)}\right) \otimes y_{j}\left(\omega_{q-2}\right)_{j}\right) .
\end{aligned}
$$

The first term on the right hand side of (9) gives the following:

$$
\begin{aligned}
& \mu D\left(\mathbf{d}\left(x d_{0} z^{(1)}\right) \otimes \omega_{q-2}\right)=\sum_{j=1}^{q-1} \mathbf{d}\left(x \mathrm{Sq}^{2}\left(y_{j}\right)+x^{2} y_{j}\right)\left(\omega_{q-1}\right)_{j}= \\
& \mathbf{d} x \sum_{j=1}^{q-1} \mathrm{Sq}^{2}\left(y_{j}\right)\left(\omega_{q-1}\right)_{j}+x \operatorname{Sq}^{2}\left(\omega_{q-1}\right)+(q-1) x^{2} \omega_{q-1} .
\end{aligned}
$$

By the formula for the shuffle map $D$ the second term on the right hand side of (91) equals

$$
\begin{gathered}
\mathbf{d} x \sum_{j=1}^{q-2} \sum_{k=1}^{q-1}\left(\mathrm{Sq}^{2}\left(\mathbf{d} y_{k}\right)+x \mathbf{d} y_{k}\right) s_{k-1}\left(y_{j}\left(\omega_{q-2}\right)_{j}\right)= \\
\mathbf{d} x \sum_{j=1}^{q-2} \sum_{k=1}^{j}\left(\mathrm{Sq}^{2}\left(\mathbf{d} y_{k}\right)+x \mathbf{d} y_{k}\right) y_{j+1}\left(\omega_{q-1}\right)_{k, j+1} \\
+\mathbf{d} x \sum_{j=1}^{q-2} \sum_{k=j+1}^{q-1}\left(\mathrm{Sq}^{2}\left(\mathbf{d} y_{k}\right)+x \mathbf{d} y_{k}\right) y_{j}\left(\omega_{q-1}\right)_{j, k} .
\end{gathered}
$$

After performing the substitution $j^{\prime}=j+1$ in the first sum on the right hand side we see that this equals

$$
\begin{aligned}
& x \mathbf{d} x \sum_{j=2}^{q-1}(j-1) y_{j}\left(\omega_{q-1}\right)_{j}+x \mathbf{d} x \sum_{j=1}^{q-2}(q-1-j) y_{j}\left(\omega_{q-1}\right)_{j} \\
+ & \mathbf{d} x \sum_{j=2}^{q-1} \sum_{k=1}^{j-1} y_{j} \operatorname{Sq}^{2}\left(\mathbf{d} y_{k}\right)\left(\omega_{q-1}\right)_{k, j}+\mathbf{d} x \sum_{j=1}^{q-2} \sum_{k=j+1}^{q-1} y_{j} \operatorname{Sq}^{2}\left(\mathbf{d} y_{k}\right)\left(\omega_{q-1}\right)_{j, k} .
\end{aligned}
$$

By a final reduction we conclude that the second term on the right hand side of (9) equals

$$
\begin{aligned}
& (q-2) x \mathbf{d} x \sum_{j=1}^{q-1} y_{j}\left(\omega_{q-1}\right)_{j} \\
+ & \mathbf{d} x \sum_{j=1}^{q-1} y_{j}\left(\sum_{k=1}^{j-1} \operatorname{Sq}^{2}\left(\mathbf{d} y_{k}\right)\left(\omega_{q-1}\right)_{k, j}+\sum_{k=j+1}^{q-1} \operatorname{Sq}^{2}\left(\mathbf{d} y_{k}\right)\left(\omega_{q-1}\right)_{j, k}\right)
\end{aligned}
$$


Since the sum of (10) and (11) equals (8) we are done.

When $n=1,|x| \geq 1$ we have that $T_{n}(x)=\Lambda(x)$ which is an object in $\mathcal{K}$ with trivial $\mathcal{A}$-action.

Proposition 4.5. The $\mathcal{A}$-action on $H_{*}(\Lambda(x) ; \bar{\Omega})$ is trivial when $|x| \geq 2$.

Proof. Put $m=|x|$. We have computed $H_{*}(\Lambda(x) ; \bar{\Omega}) \cong \Lambda(x) \otimes \Lambda(\mathbf{d} x) \otimes \Gamma[\omega]$ where $\|x\|=(m, 0),\|\mathbf{d} x\|=(m-1,0)$ and $\left\|\gamma_{q}(\omega)\right\|=(-q, q(2 m-1))$.

Because of the bigrading the only possible non zero squares on $\gamma_{q}(\omega)$ are $\mathrm{Sq}^{m-1} \gamma_{q}(\omega), \mathrm{Sq}^{m} \gamma_{q}(\omega), \mathrm{Sq}^{2 m-1} \gamma_{q}(\omega)$ which might equal $\mathbf{d} x \gamma_{q}(\omega), x \gamma_{q}(\omega)$, $x \mathbf{d} x \gamma_{q}(\omega)$ respectively. Recall that $S q^{r}$ is decomposable unless $r=2^{s}, s \geq 0$. So the result follows for all $m$ except for $m=2^{k}$ and $m=2^{k}+1$ with $k \geq 1$. When $m=2^{k}$ the result is a special case of Theorem 4.2.

Assume that $m=2^{k}+1, k \geq 1$. Here we must show that $\operatorname{Sq}^{m-1} \gamma_{q}(\omega)=0$. By the homotopy operations and algebra structure we see that it suffices to show that $\mathrm{Sq}^{m-1} \gamma_{1}(\omega)=0$ and $\mathrm{Sq}^{m-1} \gamma_{2}(\omega)=0$.

Let $R_{\bullet}^{\mathcal{K}}$ be a simplicial resolution of $\Lambda(x)$ in $s \mathcal{K}$ such that $R_{0}^{\mathcal{K}}$ is the free object in $\mathcal{K}$ on the generator $x$ (thus $R_{0}^{\mathcal{K}} \cong H^{*}\left(K\left(\mathbb{F}_{2}, m\right)\right)$ ). We have a weak equivalence of simplicial algebras $R_{\bullet} \rightarrow R_{\bullet}^{\mathcal{K}}$. Let $y_{1} \in R_{\bullet}^{\mathcal{K}}$ denote the image of $y_{1} \in R_{\bullet}$. We have $d_{0} y_{1}=x^{2}$ and $d_{1} y_{1}=0$. Since $\pi_{0}\left(R_{\bullet}^{\mathcal{K}}\right)=\Lambda(x)$ there are elements $w^{(i)} \in R_{1}^{\mathcal{K}}$ for $1 \leq i \leq m-1$ such that $d_{0} w^{(i)}=\mathrm{Sq}^{i} x$ and $d_{1} w^{(i)}=0$.

But $d_{0}\left(\mathrm{Sq}^{2 i} y_{1}+\left(w^{(i)}\right)^{2}\right)=\mathrm{Sq}^{2 i}\left(x^{2}\right)+\left(\mathrm{Sq}^{i} x\right)^{2}=0$ and $d_{0}\left(\mathrm{Sq}^{2 i+1} y_{1}\right)=$ $\mathrm{Sq}^{2 i+1}\left(x^{2}\right)=0$ so there exist elements $z^{(t)} \in \mathcal{R}_{2}^{\mathcal{K}}$ for $1 \leq t \leq 2 m-1$ with $d_{j} z^{(t)}=0$ for $j=1,2$ and $d_{0} z^{(2 i)}=\mathrm{Sq}^{2 i} y_{1}+\left(w^{(i)}\right)^{2}, d_{0} z^{(2 i+1)}=\mathrm{Sq}^{2 i+1} y_{1}$.

In $R_{\bullet}^{\mathcal{K}}$ we have $\mathbf{d} z^{(t)}$ with $d_{j} \mathbf{d} z^{(t)}=0$ and $d_{0} \mathbf{d} z^{(t)}=\mathrm{Sq}^{t} \mathbf{d} y_{1}$. Thus $\mathrm{Sq}^{m-1} \gamma_{1}(\omega)=0$. For $\gamma_{2}(\omega)$ note that

$$
\mathrm{Sq}^{2^{k}}\left(\mathbf{d} y_{1} \mathbf{d} y_{2}\right)=\sum_{t=0}^{2^{k-1}-1} m\left(d_{0} \mathbf{d} z^{(t)}, d_{0} \mathbf{d} z^{(m-t)}\right)+q\left(d_{0} \mathbf{d} z^{\left(2^{k-1}\right)}\right)
$$

so the result follows by Lemma 3.2 and Lemma 3.3

\section{The results}

Recall that if $X$ is a space with $H^{*} X=\mathbb{F}_{2}[x] /\left(x^{n+1}\right)$ where $n \geq 2$ then $|x|=2^{k}$ for $k=0,1,2$ or $|x|=8$ and $n=2$ A. Examples are the projective spaces $\mathbb{R} \mathrm{P}^{n}, \mathbb{C} \mathrm{P}^{n}, \mathbb{H} \mathrm{P}^{n}$ and the Cayley projective plane $\mathbf{C a} \mathrm{P}^{2}$, but there are other homotopy types of spaces with these cohomology rings. For $n=1$ there is a space $X$ with $H^{*} X=\Lambda(x)$ for any positive value of $|x|$. The spheres $S^{m}$ for $m \geq 1$ are examples of such spaces. 
When $n$ is odd one cannot determine the operation $\mathrm{Sq}^{1}$ by our spectral sequence. For the projective spaces and spheres this information follows from the results in $[\mathrm{Z}$.

Proposition 5.1. Consider the operation $\mathrm{Sq}^{1}: H^{t}(\Lambda X) \rightarrow H^{t+1}(\Lambda X)$.

1) $X=\mathbb{C P}^{n}, n \geq 1$ : If $n \neq 1 \bmod 4$ then all $\mathrm{Sq}^{1}=0$. If $n=1 \bmod$ 4 then the only non vanishing $\mathrm{Sq}^{1}$ 's appear when $t \in\{2 n k \mid k \geq 1\}$ and here one has $\mathrm{Sq}^{1}=(0, i d): \mathbb{F}_{2} \oplus \mathbb{F}_{2} \rightarrow \mathbb{F}_{2} \oplus \mathbb{F}_{2}$.

2) $X=\mathbb{H} \mathrm{P}^{n}, n \geq 1$ : If $n \neq 1 \bmod 4$ then all $\mathrm{Sq}^{1}=0$. If $n=1 \bmod$ 4 then the only non vanishing $\mathrm{Sq}^{1}$ 's appear when $t \in\{(4 n+2) k \mid k \geq 1\}$ and here $\mathrm{Sq}^{1}=i d: \mathbb{F}_{2} \rightarrow \mathbb{F}_{2}$.

3) $X=S^{m}, m \geq 3$ : If $m$ is odd then all $\mathrm{Sq}^{1}=0$. If $m$ is even then the only non vanishing $\mathrm{Sq}^{1}$ 's appear when $t \in\{2 k(m-1) \mid k \geq 1\}$ and here $\mathrm{Sq}^{1}=i d: \mathbb{F}_{2} \rightarrow \mathbb{F}_{2}$.

Remark 5.2. In 3) we do not mention the case $m=2$. This case is however included in 1) since $S^{2}=\mathbb{C P}^{1}$.

Proof. The homology groups of $\Lambda S^{m}, \Lambda \mathbb{C} \mathrm{P}^{n}$ and $\Lambda \mathbb{H} \mathrm{P}^{n}$ with $\mathbb{Z}$-coefficients are listed in $[\mathrm{Z}$ ] on page 21. By universal coefficients one finds the cohomology groups with coefficients in $\mathbb{Z} / 2$ and $\mathbb{Z} / 4$. The result follows by the long exact coefficient sequence associated with $0 \rightarrow \mathbb{Z} / 2 \rightarrow \mathbb{Z} / 4 \rightarrow \mathbb{Z} / 2 \rightarrow 0$.

Theorem 5.3. Assume that $X$ is a 1-connected space with cohomology algebra $H^{*} X=\mathbb{F}_{2}[x] /\left(x^{n+1}\right)$ where $n$ is a positive integer. Put $m=|x|$ and recall that when $n=1, n=2, n \geq 3$ then $m \in \mathbb{Z}_{+}, m \in\{2,4,8\}, m \in\{2,4\}$ respectively.

1) Assume that $n$ is even. Then there is an isomorphism

$$
H^{*}(\Lambda X) \cong \mathbb{F}_{2} \oplus \mathbb{F}_{2}[x] /\left(x^{n}\right)<a_{q}, b_{q} \mid q \geq 0>
$$

where $|x|=m,\left|a_{q}\right|=q m(n+1)-2 q+m-1$ and $\left|b_{q}\right|=q m(n+1)-2 q+m$. The algebra structure is given by the relations

$$
a_{p} a_{q}=0, \quad b_{p} b_{q}=\left(\begin{array}{c}
p+q \\
p
\end{array}\right) x b_{p+q}, \quad a_{p} b_{q}=\left(\begin{array}{c}
p+q \\
p
\end{array}\right) x a_{p+q} .
$$

The Steenrod algebra structure is given as follows where $i, j, q \geq 0$ :

$$
\begin{aligned}
\mathrm{Sq}^{m i}\left(x^{j} a_{q}\right) & =\left(\begin{array}{c}
q(n+1)+j \\
i
\end{array}\right) x^{i+j} a_{q}, \\
\mathrm{Sq}^{m i}\left(x^{j} b_{q}\right) & =\left(\begin{array}{c}
q(n+1)+1+j \\
i
\end{array}\right) x^{i+j} b_{q}
\end{aligned}
$$


and $\mathrm{Sq}^{t} z=0$ for all $z \in H^{*}(\Lambda X)$ when $t \neq 0 \bmod m$.

2) Assume instead that $n$ is odd. Then there is an isomorphism of algebras

$$
H^{*}(\Lambda X) \cong \mathbb{F}_{2}[x] /\left(x^{n+1}\right) \otimes \Lambda(\mathbf{d} x) \otimes \Gamma[\omega]
$$

where $|x|=m,|\mathbf{d} x|=m-1,\left|\gamma_{q}(\omega)\right|=q m(n+1)-2 q$. The Steenrod algebra action satisfies the following for $i, j, q \geq 0$ and $\epsilon=0,1$ :

$$
\mathrm{Sq}^{m i}\left(x^{j}(\mathbf{d} x)^{\epsilon} \gamma_{q}(\omega)\right)=\left(\begin{array}{c}
q(n+1)+j \\
i
\end{array}\right) x^{i+j}(\mathbf{d} x)^{\epsilon} \gamma_{q}(\omega)
$$

If $t \neq 0$ mod $m$ then $\mathrm{Sq}^{t} z=0$ for all $z \in H^{*}(\Lambda X)$ unless $z=\gamma_{q}(\omega)$ for a $q \geq 1$ and $t=1$. Here $\mathrm{Sq}^{1} \gamma_{q}(\omega)=c_{q} x^{n} \mathbf{d} x \gamma_{q-1}(\omega)$ for a constant $c_{q} \in \mathbb{F}_{2}$. If $X=\mathbb{C} \mathrm{P}^{n}$ or $X=\mathbb{H} \mathrm{P}^{n}$ then $c_{q}=1$ for $n=1 \bmod 4$ and $c_{q}=0$ for $n=3$ $\bmod 4$, for all $q \geq 1$. If $X=S^{m}, m \geq 2$ then $c_{q}=0$ for $m$ odd and $c_{q}=1$ for $m$ is even, for all $q \geq 1$.

Proof. The distribution of zero's in the $E_{2}$ therm shows that all differentials are trivial. So the spectral sequence collapse and $E_{2}=E_{\infty}$. When $n$ is even, there is a unique representative in $H^{*}(\Lambda X)$ for each element in $E_{\infty}$ and the result follows easily.

Assume that $n$ is odd. We have a filtration of $H^{*}=H^{*}(\Lambda X)$ as follows:

$$
H^{*} \supseteq \cdots \supseteq F^{-2} H^{*} \supseteq F^{-1} H^{*} \supseteq F^{0} H^{*} \supseteq F^{1} H^{*}=0
$$

with $F^{-p} H^{q-p} / F^{-p+1} H^{q-p} \cong E_{\infty}^{-p, q}$. Define an algebra map

$$
\phi: T_{n}(x) \otimes \Lambda(\mathbf{d} x) \otimes \Gamma[\omega] \rightarrow H^{*}
$$

by $x \mapsto x, \mathbf{d} x \mapsto \mathbf{d} x$ and $\gamma_{i}(\omega) \mapsto \gamma_{i}^{\prime}$ where $x$ and $\mathbf{d} x$ are the unique representatives for $x$ and $\mathbf{d} x$ in $E_{\infty}$ and $\gamma_{i}^{\prime}$ is one of the two representatives for $\gamma_{i}(\omega)$ in $E_{\infty}$. We must check that $\phi$ is well defined.

Since $\Gamma[\omega] \cong \Lambda\left(\gamma_{q}(\omega) \mid q=2^{j}, j \geq 0\right)$ it suffices to see that $\left(\gamma_{q}^{\prime}\right)^{2}=0$ when $q$ is a power of two. From the $E_{\infty}$-term we find that

$$
\begin{aligned}
\left(\gamma_{q}^{\prime}\right)^{2}=\mathrm{Sq}^{q(m(n+1)-2)}\left(\gamma_{q}^{\prime}\right) & =\left(\begin{array}{c}
m q(n+1) \\
m q(n+1)-2 q
\end{array}\right) x^{q(n+1)-2 q / m} \gamma_{q}^{\prime} \\
& =\left(\begin{array}{c}
m(n+1) q \\
2 q
\end{array}\right) x^{q(n+1)-2 q / m} \gamma_{q}^{\prime}=0
\end{aligned}
$$

So $\phi$ is well defined. We can now define a filtration on its domain space such that $\phi$ becomes a map of filtered rings. Since the associated map of graded objects is an isomorphism, $\phi$ is an isomorphism.

It follows directly from the $E_{\infty}$ term and Proposition 5.1 that the Steenrod algebra action is as stated. 


\section{A Thom space interpretation}

Let $M^{d}$ be a closed, connected and oriented smooth manifold without boundary. We denote the dual cohomology class of the fundamental class $[M] \in$ $H_{d}(M ; \mathbb{Z})$ by $u_{0}$. Let $\tau \rightarrow M$ be the tangent bundle with Thom space $T h(\tau)$ and Thom class $u_{\tau} \in H^{d}(M ; \mathbb{Z})$. The zero section defines a map $s_{0}: M \rightarrow T h(\tau)$. Recall that $s_{0}^{*}: H^{d}(T h(\tau) ; \mathbb{Z}) \rightarrow H^{d}(M ; \mathbb{Z})$ is given by multiplication with the Euler characteristics

$$
s_{0}^{*}\left(u_{\tau}\right)=\chi(M) u_{0} .
$$

Consider the $q$ fold Whitney sum $q \tau=\tau \oplus \cdots \oplus \tau$. We view $q \tau$ as a sub bundle of $(q+1) \tau$ by the identity on the first $q$ summands and zero on the last summand. Thus we have an inclusion $s_{q}: T h(q \tau) \rightarrow T h((q+1) \tau)$.

Lemma 6.1. The map $s_{q}^{*}: H^{(q+1) d}(T h((q+1) \tau) ; \mathbb{Z}) \rightarrow H^{(q+1) d}(T h(q \tau) ; \mathbb{Z})$ is given by multiplication with the Euler characteristics

$$
s_{q}^{*}\left(u_{(q+1) \tau}\right)=\chi(M) u_{q \tau} u_{0} .
$$

Proof. The Whitney sum $(q+1) \tau$ is the pullback of the product bundle $\tau^{\times(q+1)} \rightarrow M^{\times(q+1)}$ along the diagonal $\Delta_{q+1}: M \rightarrow M^{\times(q+1)}$.

We write $\epsilon^{0} \rightarrow M$ for the trivial 0-dimensional vector bundle over $M$. This bundle simply means the identity map $M \rightarrow M$ and its Thom space is $M$ with a disjoint base point $T h\left(\epsilon^{0}\right)=M_{+}$.

Form the pullback of $\tau^{\times q} \rightarrow M^{\times q}$ along a composite map as follows:

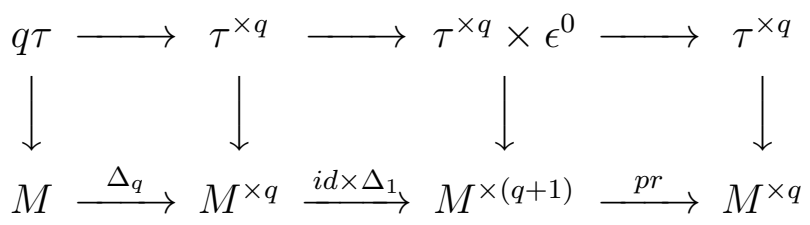

where the projection $p r$ is on the first $q$ factors. The pulback of $\tau^{\times q}$ along $p r$ equals $\tau^{\times q} \times \epsilon^{0}$. The further pulback of this vector bundle is as stated in the diagram since $p r \circ\left(i d \times \Delta_{1}\right)=i d$ and $\left(i d \times \Delta_{1}\right) \circ \Delta_{q}=\Delta_{q+1}$.

The vector bundle $\tau^{\times q} \times \epsilon^{0}$ is a sub bundle of $\tau^{\times(q+1)}$ by the map $i d \times s_{0}$. Furthermore $\tau^{\times q} \times \epsilon^{0} \subset \tau^{\times(q+1)}$ pulls back to $q \tau \subset(q+1) \tau$ along $\Delta^{q+1}$. So we get a commutative diagram as follows (with $\mathbb{Z}$-coefficients):

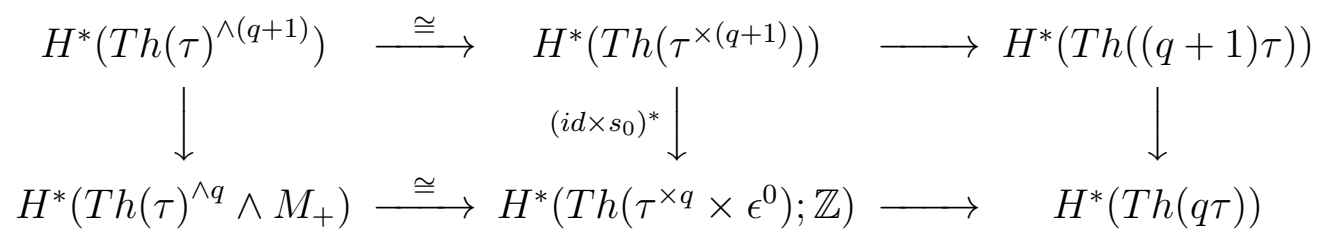


The Thom class for $\tau^{\times(q+1)}$ is $u_{\tau}^{\wedge(q+1)}$ and it maps to the Thom class $u_{(q+1) \tau}$ by the upper horizontal map. But $\left(i d \times s_{0}\right)^{*}\left(u_{\tau}^{\wedge(q+1)}\right)=u_{\tau}^{\wedge q} \wedge \chi(M) u_{0}$ so the result follows by the Thom isomorphism for the lower horizontal map.

Definition 6.2. Let $C_{q}(M)$ denote the cofiber of $s_{q}$ :

$$
T h(q \tau) \rightarrow T h((q+1) \tau) \rightarrow C_{q}(M)
$$

Proposition 6.3. Let $M^{d}$ be a compact, connected smooth manifold without boundary and of dimension $d \geq 2$. Assume that $H_{1}(M ; \mathbb{Z})=0$ and that $H_{d-1}(M ; \mathbb{Z})$ is torsion free. Assume also that the Euler characteristics $\chi(M) \neq 0$. Put $T_{q}=T h(q \tau), C_{q}=C_{q}(M)$ and write $\phi$ for the Thom isomorphism and $\partial$ for the connecting homomorphism.

1) $\mathbb{Z}$-coefficients: There are isomorphisms

$$
\begin{array}{rlrl}
\widetilde{H}_{k}\left(C_{q} ; \mathbb{Z}\right) \stackrel{\partial_{*}}{\cong} \widetilde{H}_{k-1}\left(T_{q} ; \mathbb{Z}\right) \stackrel{\phi_{*}}{\cong} H_{k-1-q d}(M ; \mathbb{Z}) & , \quad k<(q+1) d \\
\widetilde{H}_{k}\left(C_{q} ; \mathbb{Z}\right) \cong \mathbb{Z} / \chi(M) & , \quad k=(q+1) d \\
\widetilde{H}_{k}\left(C_{q} ; \mathbb{Z}\right) & \overleftarrow{\cong} \widetilde{H}_{k}\left(T_{q+1} ; \mathbb{Z}\right) \stackrel{\phi_{*}}{\cong} H_{k-(q+1) d}(M ; \mathbb{Z}) & , \quad k>(q+1) d .
\end{array}
$$

2) $\mathbb{F}_{2}$-coefficients: There are isomorphisms

$$
\begin{aligned}
& \widetilde{H}^{k}\left(C_{q}\right) \stackrel{\partial^{*}}{\cong} \widetilde{H}^{k-1}\left(T_{q}\right) \stackrel{\phi}{\cong} H^{k-1-q d}(M) \quad, \quad k<(q+1) d \\
& \widetilde{H}^{k}\left(C_{q}\right) \underset{\cong}{\cong} \widetilde{H}^{k}\left(T_{q+1}\right) \stackrel{\phi}{\cong} H^{k-(q+1) d}(M) \quad, \quad k>(q+1) d+1 .
\end{aligned}
$$

If $\chi(M)$ is odd then $\widetilde{H}^{(q+1) d}\left(C_{q}\right)=\widetilde{H}^{(q+1) d+1}\left(C_{q}\right)=0$. If $\chi(M)$ is even then

$$
\widetilde{H}^{(q+1) d}\left(C_{q}\right) \underset{\cong}{\cong} \widetilde{H}^{(q+1) d}\left(T_{q+1}\right) \quad, \quad \widetilde{H}^{(q+1) d+1}\left(C_{q}\right) \stackrel{\partial^{*}}{\cong} \widetilde{H}^{(q+1) d}\left(T_{q}\right) .
$$

The operation $\mathrm{Sq}^{1}: \widetilde{H}^{(q+1) d}\left(C_{q}\right) \rightarrow \widetilde{H}^{(q+1) d+1}\left(C_{q}\right)$ is the identity map id : $\mathbb{F}_{2} \rightarrow \mathbb{F}_{2}$ when $\chi(M)=2 \bmod 4$ and the trivial map $0: \mathbb{F}_{2} \rightarrow \mathbb{F}_{2}$ when $\chi(M)=0 \bmod 4$.

Proof. By universal coefficients $H^{1}\left(M ; \mathbb{F}_{2}\right)=0$ so $M$ is oriented. Poincaré duality gives that $H^{d-1}(M ; \mathbb{Z})=0$. So we have that $H_{d-2}(M ; \mathbb{Z})$ is torsion free and $H_{d-1}(M ; \mathbb{Z})=0$ by universal coefficients. It follows that $H^{d-1}\left(M ; \mathbb{F}_{2}\right)=0$.

The long exact (co)homology sequence and the Thom isomorphism gives the results except for the statement regarding $\mathrm{Sq}^{1}$. By universal coefficients one can determine the cohomology groups with $\mathbb{Z} / 4$-coefficients. The long exact coefficient sequence associated with $0 \rightarrow \mathbb{Z} / 2 \rightarrow \mathbb{Z} / 4 \rightarrow \mathbb{Z} / 2 \rightarrow 0$ then determines $\mathrm{Sq}^{1}$ since it agrees with the Bockstein homomorphism. 
Theorem 6.4. Let $M$ be a complex or quaternion projective space $\mathbb{C} \mathrm{P}^{n}$, $\mathbb{H} \mathrm{P}^{n}, n \geq 1$ the Cayley projective plane $\mathbf{C a} \mathrm{P}^{2}$ or a sphere $S^{m}, m \geq 2$. Let $r(M)$ denote the degree of the algebra generator for $H^{*}\left(M ; \mathbb{F}_{2}\right)$. Define the pointed space $C T(M)$ by

$$
C T(M)=M_{+} \vee \bigvee_{q \geq 0} \Sigma^{(r(M)-2)(q+1)} C_{q}(M) .
$$

Then the suspension spectra $\Sigma^{\infty}(\Lambda M)_{+}$and $\Sigma^{\infty} C T(M)$ have isomorphic homology groups with $\mathbb{Z}$-coefficients. Furthermore the cohomology groups with $\mathbb{F}_{2}$-coefficients are isomorphic modules over the Steenrod algebra. For the spheres we have a homotopy equivalence $\Sigma^{\infty}\left(\Lambda S^{m}\right)_{+} \simeq \Sigma^{\infty} C T\left(S^{m}\right)$.

Proof. We first prove the statement regarding the projective spaces and $\mathbb{Z}$ coefficients. Let $\mathbb{K}=\mathbb{C}, \mathbb{H}, \mathbf{C a}$ and put $r=r(M)=\operatorname{dim}_{\mathbb{R}} \mathbb{K}$.

We use Proposition 6.3 to compute the stable homology of $C T(M)$. The non vanishing groups $\widetilde{H}_{k}\left(C_{q}\left(\mathbb{K} \mathrm{P}^{n}\right) ; \mathbb{Z}\right)$ equal $\mathbb{Z}$ for

$$
k \in\{q d+1+j r,(q+1) d+(j+1) r \mid 0 \leq j \leq n-1\},
$$

and $\mathbb{Z} /(n+1)$ for $k=(q+1) d$. Thus $\widetilde{H}_{k}\left(\Sigma^{(r-2)(q+1)} C_{q}\left(\mathbb{K} \mathrm{P}^{n}\right) ; \mathbb{Z}\right)$ equals $\mathbb{Z}$ for $k \in A(r, q) \cup B(r, q)$ and $\mathbb{Z} /(n+1)$ for $k=((n+1) r-2)(q+1)$ and 0 otherwise where

$$
\begin{aligned}
& A(r, q)=\{((n+1) r-2) q+r j+r-1 \mid 0 \leq j \leq n-1\}, \\
& B(r, q)=\{((n+1) r-2)(q+1)+r(j+1) \mid 0 \leq j \leq n-1\} .
\end{aligned}
$$

Finally, $H_{k}\left(\mathbb{K} \mathrm{P}^{n} ; \mathbb{Z}\right)=\mathbb{Z}$ for $k \in C(r)$ where $C(r)=\{r i \mid 0 \leq i \leq n\}$ and zero otherwise.

For $\mathbb{K}=\mathbb{C}, r=2$ we have that $C(2) \cup A(2,0)=\{0, \ldots, 2 n\}$ and that $B(2, q-1) \cup A(2, q)=\{2 n q+1, \ldots, 2 n(q+1)\}$ for $q \geq 1$. So the group $\widetilde{H}_{k}\left(C T\left(\mathbb{C} \mathrm{P}^{n}\right) ; \mathbb{Z}\right)$ equals $\mathbb{Z} \oplus \mathbb{Z} /(n+1)$ for $k=2 n m, m \geq 1$ and $\mathbb{Z}$ otherwise. For $H_{k}\left(\Lambda \mathbb{C} \mathrm{P}^{n} ; \mathbb{Z}\right)$ we have the same by the table in $[\mathbf{Z}$.

For $\mathbb{K}=\mathbb{H}, r=4$ the group $\widetilde{H}_{k}\left(C T\left(\mathbb{H} \mathrm{P}^{n}\right) ; \mathbb{Z}\right)$ equals $\mathbb{Z}$ when $k$ belongs to one of the sets

$$
\begin{aligned}
& C(4) \cup \cup_{q \geq 0} B(4, q)=\{0\} \cup\{2 m(2 n+1)+4 l \mid m \geq 1,1 \leq l \leq n\}, \\
& \cup_{q \geq 0} A(4, q)=\{2 m(2 n+1)+4 l-4 n+1 \mid m \geq 1,0 \leq l \leq n-1\},
\end{aligned}
$$

and $\mathbb{Z} /(n+1)$ when $k \in\{2 m(2 n+1) \mid m \geq 1\}$. Otherwise the group vanish. For $H_{k}\left(\Lambda \mathbb{H} \mathrm{P}^{n} ; \mathbb{Z}\right)$ we have the same by $[\mathbf{Z}$.

For $\mathbb{K}=\mathbf{C a}, r=8, n=2$ the group $\widetilde{H}_{k}\left(C T\left(\mathbf{C a P}^{2}\right) ; \mathbb{Z}\right)$ equals $\mathbb{Z}$ for $k \in\{0,8,16\} \cup\{22 m-15,22 m-7,22 m+8,22 m+16 \mid m \geq 1\}$ and a $\mathbb{Z} / 3$ 
for $k \in\{22 m \mid m \geq 1\}$. The list in [Z] differs here by having zero's in degrees $0,8,16$ and $22 m-15, m \geq 1$ instead of $\mathbb{Z}$ 's.

This is because there is an error in Ziller's final table of $H_{*}\left(\Lambda \mathbf{C a} \mathrm{P}^{2} ; \mathbb{Z}\right)$. His result is the same as ours when one corrects this error as we will now see. By Z Theorem 8 one has

$$
H_{*}\left(\Lambda \mathbf{C a ~}^{2} ; \mathbb{Z}\right) \cong \bigoplus_{B} H_{*-\lambda(B)}(B ; \mathbb{Z})
$$

where the sum is over all critical sub manifolds $B \subset \Lambda \mathbf{C a P}^{2}$ and $\lambda(M)$ is the index of $M$. All the critical sub manifolds are diffeomorphic to the sphere bundle $T_{1} M=S(\tau)$. By the table on page 20 of $[\mathrm{Z}]$ one sees that $H_{k}\left(T_{1} \mathbf{C a ~ P}^{2} ; \mathbb{Z}\right)=\mathbb{Z}$ for $k=0,8,23,31$ and $H_{k}\left(T_{1} \mathbf{C a ~} \mathrm{P}^{2} ; \mathbb{Z}\right)=\mathbb{Z} / 3$ for $k=$ 15 and zero otherwise. The index can be found in the table on page 11: $\lambda\left(c^{m}\right)=22 m-15$.

So Ziller shows that

$$
H_{k}\left(\Lambda \mathbf{C a P}^{2} ; \mathbb{Z}\right) \cong H_{k}\left(\mathbf{C a ~} P^{2} ; \mathbb{Z}\right) \oplus \bigoplus_{m \geq 1} H_{k-22 m+15}\left(T_{1} \mathbf{C a ~ P}^{2} ; \mathbb{Z}\right)
$$

Thus one gets a $\mathbb{Z}$ when $k \in\{0,8,16\}$ or $k=22 m-15+t$ for $t \in\{0,8,23,31\}$, $m \geq 1$ and one gets a $\mathbb{Z} / 3$ when $k=22 m, m \geq 1$. This is the same as our result.

Now we prove the statements regarding $\mathbb{F}_{2}$-coefficients for the projective spaces. We use Proposition 6.3 to find the $\mathcal{A}$-action on $\widetilde{H}^{*}\left(C T\left(\mathbb{K ~ P}^{n}\right)\right)$. We have that $H^{*}\left(\mathbb{K}^{n}\right)=\mathbb{F}_{2}[x] /\left(x^{n+1}\right)$ where $|x|=r$. By [MS] page 133, Wu's theorem implies that the total Stiefel-Whitney class of $\tau$ is $w(\tau)=(1+x)^{n+1}$ and hence

$$
w(q \tau)=(1+x)^{q(n+1)}=\sum_{i=0}^{\infty}\left(\begin{array}{c}
q(n+1) \\
i
\end{array}\right) x^{i} .
$$

We have a Thom isomorphism $\phi: H^{*}\left(\mathbb{K} \mathrm{P}^{n}\right) \rightarrow \widetilde{H}^{*+r n q}(T h(q \tau))$ and the action on the fundamental class satisfies $\mathrm{Sq}^{k} u_{q \tau}=\phi\left(w_{k}(q \tau)\right)$ such that

$$
S q^{r i} u_{q}=\left(\begin{array}{c}
q(n+1) \\
i
\end{array}\right) x^{i} u_{q} \quad, \quad \mathrm{Sq}^{k} u_{q}=0 \text { if } k \neq 0 \bmod r
$$

Assume that $n$ is even. Then $\chi\left(\mathbb{K}^{n}\right)=n+1$ is odd. Proposition 6.3 shows that $\widetilde{H}^{*}\left(\Sigma^{(r-2)(q+1)} C_{q}\left(\mathbb{K}^{n}\right)\right)$ has $\mathbb{F}_{2}$-basis $\left\{a_{q}^{j}, b_{q+1}^{j} \mid 0 \leq j<n\right\}$ with

$$
\begin{aligned}
& a_{q}^{j}=\sigma^{(r-2)(q+1)} \partial^{*}\left(x^{j} u_{q \tau}\right) \quad, \quad b_{q+1}^{j}=\sigma^{(r-2)(q+1)}\left(Q^{*}\right)^{-1}\left(x^{j+1} u_{(q+1) \tau}\right), \\
& \left|a_{q}^{j}\right|=q r(n+1)-2 q+r-1+r j, \\
& \left|b_{q+1}^{j}\right|=(q+1) r(n+1)-2(q+1)+r+r j
\end{aligned}
$$


where $Q: T h((q+1) \tau) \rightarrow C_{q}\left(\mathbb{K} \mathrm{P}^{n}\right)$ denotes the quotient map. By (12) and the Cartan formula we have

$$
\begin{aligned}
& \operatorname{Sq}^{r i}\left(a_{q}^{j}\right)=\left(\begin{array}{c}
q(n+1)+j \\
i
\end{array}\right) a_{q}^{i+j}, \\
& \operatorname{Sq}^{r i}\left(b_{q+1}^{j}\right)=\left(\begin{array}{c}
(q+1)(n+1)+1+j \\
i
\end{array}\right) b_{q+1}^{i+j}
\end{aligned}
$$

where by definition $a_{q}^{t}=b_{q+1}^{t}=0$ if $t \geq n$. The operations $\mathrm{Sq}^{k}$ for $k \neq 0$ mod $r$ sends the basis elements to zero. The reason why there is no therms of the form $a_{q}^{j}$ in the formula for $\mathrm{Sq}^{t}\left(b_{q+1}^{j}\right)$ is that the degrees of such terms are to small. The result follows by comparing with Theorem 5.3.

Assume that $n$ is odd. Then $\chi\left(\mathbb{K} \mathrm{P}^{n}\right)=n+1$ is even. Proposition 6.3 shows that $\widetilde{H}^{*}\left(\Sigma^{(r-2)(q+1)} C_{q}\left(\mathbb{K}^{n}\right)\right)$ has $\mathbb{F}_{2}$-basis $\left\{c_{q}^{j}, d_{q+1}^{j} \mid 0 \leq j \leq n\right\}$ with

$$
\begin{aligned}
& c_{q}^{j}=\sigma^{(r-2)(q+1)} \partial\left(x^{j} u_{q \tau}\right) \quad, \quad d_{q+1}^{j}=\sigma^{(r-2)(q+1)} Q^{-1}\left(x^{j} u_{(q+1) \tau}\right), \\
& \left|c_{q}^{j}\right|=q r(n+1)-2 q+1+r j \\
& \left|d_{q+1}^{j}\right|=(q+1) r(n+1)-2(q+1)+r j
\end{aligned}
$$

By (12) and the Cartan formula we have

$$
\begin{aligned}
& \operatorname{Sq}^{r i}\left(c_{q}^{j}\right)=\left(\begin{array}{c}
q(n+1)+j \\
i
\end{array}\right) c_{q}^{i+j}, \\
& \operatorname{Sq}^{r i}\left(b_{q+1}^{j}\right)=\left(\begin{array}{c}
(q+1)(n+1)+j \\
i
\end{array}\right) b_{q+1}^{i+j}
\end{aligned}
$$

where by definition $a_{q}^{t}=b_{q+1}^{t}=0$ if $t>n$. The operations $\mathrm{Sq}^{k}$ for $k \neq 0$ $\bmod r$ vanish on the basis elements except for $\mathrm{Sq}^{1} d_{q+1}^{0}$ which equals $c_{q}^{n}$ when $n=1 \bmod 4$ and zero when $n=3 \bmod 4$. Note that we cannot hit any of the classes $b_{q+1}^{j}$ by a Steenrod operation on one of the classes $a_{q}^{j}$ since $a_{q}^{j}$ is in the image of $\partial$. The result follows by comparing with Theorem 5.3. The class $c_{q}^{j}$ corresponds to $x^{j} \mathbf{d} x \gamma_{q}(\omega)$ and $d_{q+1}^{j}$ corresponds to $x^{j} \gamma_{q+1}(\omega)$.

For the spheres we can prove directly that there is a homotopy equivalence. We first identify the stable homotopy type of $C_{q}\left(S^{m}\right)$. We have $S^{m} \subseteq \mathbb{R}^{m+1}$ with trivial one dimensional normal bundle $\epsilon^{1} \rightarrow S^{m}$ and $\tau \oplus \epsilon^{1} \cong \epsilon^{m+1}$. Thus

$$
\begin{aligned}
\Sigma^{q+t} T h(q \tau) \cong & T h\left((q+t) \epsilon^{1} \oplus q \tau\right) \cong T h\left(\epsilon^{q(m+1)+t}\right) \cong \Sigma^{q(m+1)+t} S_{+}^{m} \cong \\
& S^{q(m+1)+t} \vee S^{q(m+1)+m+t} .
\end{aligned}
$$

By taking the $(q+1)$-fold suspension in Definition 6.2 we find that

$$
S^{q(m+1)+1} \vee S^{(q+1)(m+1)} \rightarrow S^{(q+1)(m+1)} \vee S^{(q+1)(m+1)+m} \rightarrow \Sigma^{q+1} C_{q}\left(S^{m}\right)
$$


Any map $S^{r} \rightarrow S^{s}$ is null homotopic when $r<s$ and a map $S^{r} \rightarrow S^{r}$ is determined, up to homotopy, by its degree. By Proposition 6.1 we have that the degree of the above self map of $S^{(q+1)(m+1)}$ is $\chi\left(S^{m}\right)$ which is 0 for m odd and 2 for $m$ even. So $\Sigma^{q+1} C_{q}\left(S^{m}\right)$ is homotopy equivalent to the following wedge of spheres and Moore spaces:

$$
\begin{cases}S^{(q+1)(m+1)} \vee S^{(q+1)(m+1)+m} \vee S^{q(m+1)+2} \vee S^{(q+1)(m+1)+1} & , m \text { odd } \\ M(\mathbb{Z} / 2,(q+1)(m+1)) \vee S^{(q+1)(m+1)+m} \vee S^{q(m+1)+2} & , m \text { even. }\end{cases}
$$

Thus $\Sigma^{(m-2)(q+1)} C_{q}\left(S^{m}\right)$ is stably equivalent to

$$
\begin{cases}S^{2(q+1)(m-1)} \vee S^{2(q+1)(m-1)+m} \vee S^{(2 q+1)(m-1)} \vee S^{2(q+1)(m-1)+1} & , m \text { odd } \\ M(\mathbb{Z} / 2,2(q+1)(m-1)) \vee S^{2(q+1)(m-1)+m} \vee S^{(2 q+1)(m-1)} & , m \text { even. }\end{cases}
$$

So we have determined the stable homotopy type of $C T\left(S^{m}\right)$.

On the other hand the results in $[\mathrm{BM}], \mathrm{CC}]$ give a stable decomposition of $\Lambda \Sigma Y$ where $Y$ is a based connected space. Let $C_{k} \subseteq S^{1}$ denote the cyclic group of order $k$ and put $D_{k}(Y)=S_{+}^{1} \wedge_{C_{k}} Y^{\wedge k}$. Then there is a stable equivalence

$$
\Lambda \Sigma Y \simeq \bigvee_{k \geq 1} D_{k}(Y)
$$

We use this result for $Y=S^{m-1}$. Here each $D_{k}\left(S^{m-1}\right)$ decomposes further.

The cofiber of the inclusion $i: C_{k+} \subseteq S_{+}^{1}$ is a $k$-fold wedge of circles. We have an associated Barrat-Puppe sequence

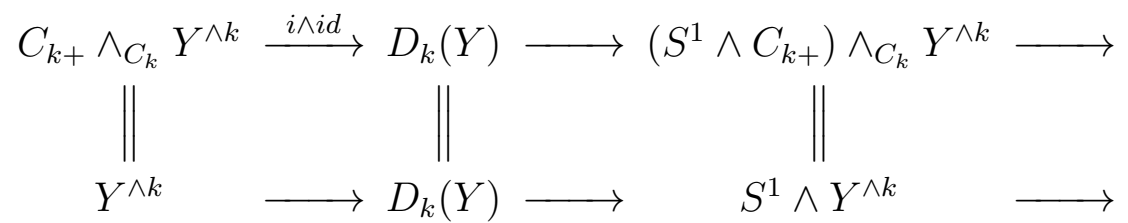

So for $Y=S^{m-1}$ we find

$$
S^{(m-1) k} \rightarrow D_{k}\left(S^{m-1}\right) \rightarrow S^{(m-1) k+1} \stackrel{\partial_{m-1}}{\longrightarrow} S^{(m-1) k+1} \rightarrow \Sigma D_{k}\left(S^{m-1}\right) \rightarrow \ldots
$$

By analyzing the Barrat-Puppe sequence

$$
C_{k+} \stackrel{i}{\rightarrow} S_{+}^{1} \rightarrow S^{1} \wedge C_{k+} \stackrel{\partial}{\rightarrow} S^{1} \wedge C_{k+} \rightarrow S^{1} \wedge S_{+}^{1} \rightarrow \ldots
$$

via the mapping cone of $i$ we see that $\left(\partial_{m-1}\right)_{*}=i d-T_{*}$ on reduced integral homology. Shifting to integral cohomology we see that the degree of $\partial_{m-1}$ is 2 when $m-1$ is odd and $k-1$ is odd and 0 otherwise. Thus

$$
\Sigma D_{k}\left(S^{m-1}\right) \simeq \begin{cases}M(\mathbb{Z} / 2,(m-1) k+1) & , \text { for } m \text { and } k \text { even } \\ S^{(m-1) k+1} \vee S^{(m-1) k+2} & , \text { otherwise }\end{cases}
$$


So $D_{k}\left(S^{m-1}\right)$ is stably equivalent to $M(\mathbb{Z} / 2,(m-1) k)$ when $m$ and $k$ are both even and stably equivalent to $S^{(m-1) k} \vee S^{(m-1) k+1}$ otherwise. The result follows.

\section{Appendix: A property of the shuffle map}

Let $A_{\bullet}$ and $B_{\bullet}$ be simplicial modules over a commutative ring $k$. Recall that the shuffle map $D: C_{*}(A) \otimes C_{*}(B) \rightarrow C_{*}(A \otimes B)$ is defined as follows [ML] VIII.8:

$$
D_{n}(a \otimes b)=\sum_{(\mu, \nu)}(-1)^{\epsilon(\mu)} s_{\nu_{q}} \ldots s_{\nu_{1}} a \otimes s_{\mu_{p}} \ldots s_{\mu_{1}} b
$$

where $a \in A_{p}$ and $b \in B_{q}$ with $p+q=n$. The sum is taken over all $(p, q)$ shuffles $(\mu, \nu)$ cf. [ML] and the sign is given by the formula

$$
\epsilon(\mu)=\sum_{i=1}^{p}\left(\mu_{i}-(i-1)\right)
$$

We want to investigate how the shuffle map commutes with the simplicial boundary maps. Recall that

$$
d_{i} s_{j}= \begin{cases}s_{j-1} d_{i} & , i<j \\ i d & , i=j \text { or } i=j+1 \\ s_{j} d_{i-1} & , i>j+1\end{cases}
$$

Proposition 7.1. There is a commutative diagram as follows:

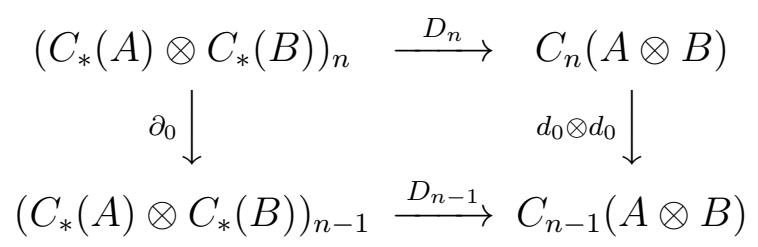

where the map $\partial_{0}$ is defined by

$\partial_{0}: A_{p} \otimes B_{q} \rightarrow\left(A_{p-1} \otimes B_{q}\right) \oplus\left(A_{p} \otimes B_{q-1}\right) ; \quad \partial_{0}=d_{0} \otimes i d+(-1)^{p} i d \otimes d_{0}$.

Here $A_{j}=B_{j}=0$ when $j<0$ by convention. When $i \geq 1$ there are $k$-linear maps $F_{t}, G_{t}:\left(C_{*}(A) \otimes C_{*}(B)\right)_{n-1} \rightarrow C_{n-1}(A \otimes B)$ for $1 \leq t \leq i$ such that

$$
\left(d_{i} \otimes d_{i}\right) \circ D_{n}=\sum_{t=1}^{i}\left(F_{t} \circ\left(d_{t} \otimes i d\right)+G_{t} \circ\left(i d \otimes d_{t}\right)\right) .
$$


Proof. We rewrite the sum defining $\left(d_{0} \otimes d_{0}\right) \circ D_{n}$ by commuting $d_{0}$ and $s_{\mu_{j}}$, using formula (14). There are two possibilities:

$$
d_{0} s_{\mu_{p}} \ldots s_{\mu_{1}}= \begin{cases}s_{\mu_{p}-1} \ldots s_{\mu_{1}-1} d_{0} & \text { if } \mu_{1} \geq 1 \\ s_{\mu_{p}-1} \ldots s_{\mu_{2}-1} & \text { if } \mu_{1}=0\end{cases}
$$

corresponding to the two cases

$$
\begin{aligned}
d_{0} s_{\nu_{q}} \ldots s_{\nu_{1}} \otimes d_{0} s_{\mu_{p}} \ldots s_{\mu_{1}}= & \begin{cases}s_{\nu_{q}-1} \ldots s_{\nu_{2}-1} \otimes s_{\mu_{p}-1} \ldots s_{\mu_{1}-1} d_{0} & \text { if } \nu_{1}=0 \\
s_{\nu_{q}-1} \ldots s_{\nu_{1}-1} d_{0} \otimes s_{\mu_{p}-1} \ldots s_{\mu_{2}-1} & \text { if } \mu_{1}=0 .\end{cases}
\end{aligned}
$$

To the shuffle $(\mu, \nu)$ of the elements in $[n-1]$, we associate a new shuffle $(\mu-1, \nu-1)$ on $[n-2]$ by the rule $(\mu-1)_{i}=\mu_{i}-1$ and $(\nu-1)_{i}=\nu_{i}-1$. There is of course the problem that $(\mu-1)_{1}$ can be negative. Actually, either $(\mu-1)_{1}=-1$ or $(\nu-1)_{1}=-1$, and we delete the corresponding element. What we have left is a genuine shuffle on $[n-2]$.

This defines a bijection between shuffles on $[n-1]$ such that $\mu_{1}=0$ and shuffles on $[n-2]$. Similarly, it defines a bijection between shuffles of $[n-1]$ element such that $\nu_{1}=0$ and shuffles on $[n-2]$ elements.

The sign of the shuffle $(\mu-1, \nu-1)$ is, according to formula (13) given by

$$
\epsilon(\mu-1)= \begin{cases}\sum_{1 \leq i \leq p-1}\left((\mu-1)_{i}-(i-1)\right)=\epsilon(\mu) & \text { if } \mu_{1}=0 \\ \sum_{1 \leq i \leq p}\left((\mu-1)_{i}-(i-1)\right)=\epsilon(\mu)-p & \text { if } \nu_{1}=0 .\end{cases}
$$

So we can express the sum defining $\left(d_{0} \otimes d_{0}\right) \circ D_{n}$ as a sum of shuffles (with sign) applied to either $\left(d_{0} \otimes i d\right)$ or $\left(i d \otimes d_{0}\right)$. Thus the diagram commutes.

Let $i \geq 1$. We use formula (14) to rewrite $d_{i} s_{\mu_{p}} \ldots s_{\mu_{1}}$, moving the $d_{i}$ toward the right. There are two possibilities.

If $\{i, i-1\} \cap\left\{\mu_{1}, \ldots, \mu_{p}\right\}=\emptyset$ we get that the element equals some $s_{\mu_{p}^{\prime}} \ldots s_{\mu_{1}^{\prime}} d_{r}$. Looking closer at the formula (14), we see that since $i \geq 1$, we have that $i \geq r \geq 1$. This means that there are maps $\alpha$ and $\beta$ such that

$$
\left(d_{i} \otimes d_{i}\right)\left(s_{\nu_{q}} \ldots s_{\nu_{1}} a \otimes s_{\mu_{p}} \ldots s_{\mu_{1}} b\right)=(\alpha \otimes \beta) \circ\left(i d \otimes d_{r}\right)(a \otimes b) .
$$

Similarly, if $\{i, i-1\} \cap\left\{\nu_{1}, \ldots, \nu_{q}\right\}=\emptyset$ there are maps $\alpha^{\prime}$ and $\beta^{\prime}$ and an integer $s$ with $i \geq s \geq 1$ such that

$$
\left(d_{i} \otimes d_{i}\right)\left(s_{\nu_{q}} \ldots s_{\nu_{1}} a \otimes s_{\mu_{p}} \ldots s_{\mu_{1}} b\right)=\left(\alpha^{\prime} \otimes \beta^{\prime}\right) \circ\left(d_{s} \otimes i d\right)(a \otimes b) .
$$

We now only have to take care of the summands of $D_{n}$ corresponding to shuffles such that either $i=\mu_{r}$ and $i-1=\nu_{s}$ for some $r, s$ or $i-1=\mu_{r}$ 
and $i=\nu_{s}$ for some $r, s$. These shuffles come in pairs: If $(\mu, \nu)$ is a shuffle with $\mu_{r}=i$ and $\nu_{s}=i-1$, then we can define a different shuffle $\left(\mu^{\prime}, \nu^{\prime}\right)$ by interchanging $\mu_{r}$ and $\nu_{s}$. Thus

$$
\mu_{t}^{\prime}= \begin{cases}\mu_{t} & \text { if } t \neq r \\ i-1 & \text { if } t=r\end{cases}
$$

Then $(\mu, \nu)$ and $\left(\mu^{\prime}, \nu^{\prime}\right)$ will according to formula (13) contribute equal terms with opposite signs in the sum computing $\left(d_{i} \otimes d_{i}\right) \circ D_{n}$. These terms cancel, and the second part of the proposition is proved.

\section{References}

[A] J. F. Adams, On the non-existence of elements of Hopf invariant one, Ann. of Math. 72 (1960), 20-104.

[BK] A.K. Bousfield \& D.M. Kan, Homotopy limits and localizations, Springer Lecture Notes in Mathematics, Vol 304(1972)

[BM] C.-F. Bödigheimer \& I. Madsen, Homotopy quotients of mapping spaces and their stable splitting, Quart. J. Math. Oxford (2), 39 (1988), 401-409.

[BO1] M. Bökstedt \& I. Ottosen, A spectral sequence for string cohomology, Copenhagen preprint (2002).

[BO2] M. Bökstedt \& I. Ottosen, An alternative approach to homotopy operations, Århus preprint (2004).

[CC] G. Carlsson \& R. Cohen, The cyclic groups and the free loop space, Comment. Math. Helv. 62 (1987), 423-449.

[CS] M. Chas \& D. Sullivan, String Topology, to appear in Annals of Math.

[D] W. G. Dwyer, Homotopy operations for simplicial commutative algebras, Trans A.M.S. 260 (1980), 421-435.

[G] P. G. Goerss, André-Quillen cohomology and the homotopy groups of mapping spaces: Understanding the $E_{2}$-term of the BousfieldKan spectral sequence, JPAA 63 (1990), 113-153.

[KY] K. Kuribayashi \& T. Yamaguchi, The cohomology algebra of certain free loop spaces, Fund. Math. 154 (1997), 57-73. 
[ML] S. MacLane, Homology, Springer 1975.

[MS] J. W. Milnor \& J. D. Stasheff, Characteristic Classes, Annals of Mathematics Studies, Princeton University Press 1974.

[T] H. Toda, Note on cohomology ring of certain spaces, Proc. Amer. Math. Soc. 14 (1963), 89-95.

[Z] W. Ziller, The free loop space of globally symmetric spaces, Invent. Math. 41 (1977), 1-22. 